\title{
Efficient computation of longest single-arm-gapped palindromes in a string
}

\author{
Shintaro Narisada ${ }^{1}$, Diptarama Hendrian ${ }^{1}$, Kazuyuki Narisawa ${ }^{1}$, Shunsuke Inenaga ${ }^{2}$, and \\ Ayumi Shinohara ${ }^{1}$ \\ ${ }^{1}$ Graduate School of Information Sciences, Tohoku University, Sendai, Japan \\ ${ }^{2}$ Department of Informatics, Kyushu University, Fukuoka, Japan
}

\begin{abstract}
In this paper, we introduce new types of approximate palindromes called single-arm-gapped palindromes (shortly SAGPs). A SAGP contains a gap in either its left or right arm, which is in the form of either $w g u c u^{R} w^{R}$ or $w u c u^{R} g w^{R}$, where $w$ and $u$ are non-empty strings, $w^{R}$ and $u^{R}$ are respectively the reversed strings of $w$ and $u, g$ is a string called a gap, and $c$ is either a single character or the empty string. Here we call $w u$ and $u^{R} w^{R}$ the arm of the SAGP, and $|u v|$ the length of the arm. We classify SAGPs into two groups: those which have $u c u^{R}$ as a maximal palindrome (type-1), and the others (type-2). We propose several algorithms to compute type-1 SAGPs with longest arms occurring in a given string, based on suffix arrays. Then, we propose a linear-time algorithm to compute all type-1 SAGPs with longest arms, based on suffix trees. Also, we show how to compute type-2 SAGPs with longest arms in linear time. We also perform some preliminary experiments to show practical performances of the proposed methods.
\end{abstract}

\section{Introduction}

A palindrome is a string that reads the same forward and backward. Discovering palindromic structures in strings is a classical and important task in combinatorics on words and string algorithmics (e.g., see [6, 12, 18, 1]). A natural extension to palindromes is to allow for a gap between the left and right arms of palindromes. Namely, a string $x$ is called a gapped palindrome if $x=w g w^{R}$ for some strings $w, g$ with $|w| \geq 1$ and $|g| \geq 0$. For instance, string abbca $\mid$ acbba is a palindrome and string abbca $\mid$ baa $\mid$ acbba is a gapped palindrome with baa as a gap. Finding gapped palindromes has applications in bioinformatics, such as finding secondary structures of RNA sequences called hairpins [13]. If we further allow for another gap inside either arm, then such a palindrome can be written as $w g_{2} u g_{1} u^{R} w^{R}$ or $w u g_{1} u^{R} g_{2} w^{R}$ for some strings $w, g_{1}, g_{2}, u$ with $|u| \geq 1,\left|g_{1}\right| \geq 0,\left|g_{2}\right| \geq 0$, and $|w| \geq 1$. These types of palindromes characterize hairpins with bulges in RNA sequences, known to occur frequently in the secondary structures of RNA sequences [21. Notice that the special case where $\left|g_{1}\right| \leq 1$ and $\left|g_{2}\right|=0$ corresponds to usual palindromes, and the special case where $\left|g_{1}\right| \geq 2$ and $\left|g_{2}\right|=0$ corresponds to gapped palindromes.

In this paper, we consider a new class of generalized palindromes where $\left|g_{1}\right| \leq 1$ and $\left|g_{2}\right| \geq 1$, i.e., palindromes with gaps only inside one of its arms. We call such palindromes as single-arm-gapped palindromes $(S A G P s)$. For instance, string abb $|\mathrm{ca}| \mathrm{cb}|\mathrm{bc}| \mathrm{bba}$ is an SAGP of this kind, taking $w=\mathrm{abb}$, $g_{1}=\varepsilon$ (the empty string), $g_{2}=\mathrm{ca}$, and $u=\mathrm{cb}$.

(C)2019. This manuscript version is made available under the CC BY-NC-ND 4.0 license

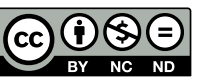


We are interested in occurrences of SAGPs as substrings of a given string $T$. For simplicity, we will concentrate on SAGPs with $\left|g_{1}\right|=0$ containing a gap in their left arms. However, slight modification of all the results proposed in this paper can easily be applied to all the other cases. For any occurrence of an SAGP $w g u u^{R} w^{R}$ beginning at position $b$ in $T$, the position $b+|w g u|-1$ is called the pivot of the occurrence of this SAGP. This paper proposes various algorithms to solve the problem of computing longest SAGPs for every pivot in a given string $T$ of length $n$. We classify longest SAGPs into two groups: those which have $u u^{R}$ as a maximal palindrome (type-1), and the others (type-2). Firstly, we show a naïve $O\left(n^{2}\right)$-time algorithm for computing type-1 longest SAGPs. Secondly, we present a simple but practical $O\left(n^{2}\right)$-time algorithm for computing type-1 longest SAGPs based on simple scans over the suffix array [19]. We also show that the running time of this algorithm can be improved by using a dynamic predecessor/successor data structure. If we employ the van Emde Boas tree [22, we achieve $O\left(\left(n+o c c_{1}\right) \log \log n\right)$-time solution, where $o c c_{1}$ is the number of type-1 longest SAGPs to output. Finally, we present an $O\left(n+o c c_{1}\right)$-time solution based on the suffix tree data structure [23. For type-2 longest SAGPs, we show an $O\left(n+o c c_{2}\right)$ time algorithm, where $\mathrm{occ}_{2}$ is the number of type-2 longest SAGPs to output. Combining the last two results, we obtain an optimal $O(n+o c c)$-time algorithm for computing all longest SAGPs, where occ is the number of outputs.

We performed preliminary experiments to compare practical performances of our algorithms for finding type-1 longest SAGPs. We compare the running time of the naïve algorithm, the $O\left(n^{2}\right)$-time suffix array based algorithm, and the improved suffix array based algorithm with several kinds of predecessor/successor data structures in the experiments.

\section{Related work}

For a fixed gap length $d$, one can find all gapped palindromes $w g w^{R}$ with $|g|=d$ in the input string $T$ of length $n$ in $O(n)$ time [13. Kolpakov and Kucherov [17] showed an $O(n+L)$-time algorithm to compute long-armed palindromes in $T$, which are gapped palindromes $w g w^{R}$ such that $|w| \geq|g|$. Here, $L$ denotes the number of outputs. They also showed how to compute, in $O(n+L)$ time, length-constrained palindromes which are gapped palindromes $w g w^{R}$ such that the gap length $|g|$ is in a predefined range. Fujishige et al. [10] proposed online algorithms to compute long-armed palindromes and length-constrained palindromes from a given string $T$. A gapped palindrome $w g w^{R}$ is an $\alpha$-gapped palindrome, if $|w g| \leq \alpha|w|$ for $\alpha \geq 1$. Gawrychowski et al. [11] showed that the maximum number of $\alpha$-gapped palindromes occurring in a string of length $n$ is at most $28 \alpha n+7 n$. Very recently, I and Köppl [14] showed an improved bound $7\left(\pi^{2} / 6+1 / 2\right) \alpha n-5 n-1$ for the maximum number of $\alpha$-gapped palindromes. Since long-armed palindromes are 2-gapped palindromes for $\alpha=2, L=O(n)$ and thus Kolpakov and Kucherov's algorithm runs in $O(n)$ time. Gawrychowski et al. [11] also proposed an $O(\alpha n)$-time algorithm to compute all $\alpha$-gapped palindromes in a given string for any predefined $\alpha \geq 1$. We emphasize that none of the above algorithms can directly be applied to computing SAGPs.

Crochemore et al. 5] proposed a linear-time algorithm to compute longest previous reverse factor array LPrF of a string $T$, where $\operatorname{LPrF}[i]$ stores the longest factor $w$ started at $i$ such that $w^{R}$ occurs at $T[1 . . i-1]$ which can be considered as a gapped palindrome. Later Dumitran et al. [8, 7] studied similar arrays that bound the length of gaps.

A preliminary version of this work appeared in [20].

\section{Preliminaries}

Let $\Sigma=\{1, \ldots, \sigma\}$ be an integer alphabet of size $\sigma$. An element of $\Sigma^{*}$ is called a string. For any string $w$, $|w|$ denotes the length of $w$. The empty string is denoted by $\varepsilon$. Let $\Sigma^{+}=\Sigma^{*} \backslash\{\varepsilon\}$. For any $1 \leq i \leq|w|$, $w[i]$ denotes the $i$-th symbol of $w$. For a string $w=x y z$, strings $x, y$, and $z$ are called a prefix, substring, 
and suffix of $w$, respectively. The substring of $w$ that begins at position $i$ and ends at position $j$ is denoted by $w[i . . j]$ for $1 \leq i \leq j \leq|w|$, i.e., $w[i . . j]=w[i] \cdots w[j]$. For $j>i$, let $w[i . . j]=\varepsilon$ for convenience. For two strings $X$ and $Y$, let $\operatorname{lcp}(X, Y)$ denote the length of the longest common prefix of $X$ and $Y$.

For any string $x$, let $x^{R}$ denote the reversed string of $x$, i.e. $x^{R}=x[|x|] \cdots x[1]$. A string $p$ is called a palindrome if $p=p^{R}$. Let $T$ be any string of length $n$. Let $p=T[b . . e]$ be a palindromic substring of $T$. The position $i=\left\lfloor\frac{b+e}{2}\right\rfloor$ is called the center of this palindromic substring $p$. The palindromic substring $p$ is said to be the maximal palindrome centered at $i$ iff there are no longer palindromes than $p$ centered at $i$, namely, $T[b-1] \neq T[e+1], b=1$, or $e=n$.

A string $x$ is called a single-arm-gapped palindrome (SAGP) if $x$ is in the form of either $w g u c u^{R} w^{R}$ or $w u c u^{R} g w^{R}$, with some non-empty strings $w, g, u \in \Sigma^{+}$and $c \in \Sigma \cup\{\varepsilon\}$. For simplicity and ease of explanations, in what follows we consider only SAGPs whose left arms contain gaps and $c=\varepsilon$, namely, those of form $w g u u^{R} w^{R}$. But our algorithms to follow can easily be modified to compute other forms of SAGPs occurring in a string as well.

Let $b$ be the beginning position of an occurrence of a SAGP $w g u u^{R} w^{R}$ in $T$, namely $T[b . . b+2|w u|+$ $|g|-1]=\operatorname{wguu}^{R} w^{R}$. The position $i=b+|w g u|-1$ is called the pivot of this occurrence of the SAGP. This position $i$ is also the center of the palindrome $u u^{R}$. An SAGP $w g u u^{R} w^{R}$ for pivot $i$ in string $T$ is represented by a quadruple $(i,|w|,|g|,|u|)$ of integers. In what follows, we will identify the quadruple $(i,|w|,|g|,|u|)$ with the corresponding SAGP $w g u u^{R} w^{R}$ for pivot $i$.

For any SAGP $x=w g u u^{R} w^{R}$, let $\operatorname{armlen}(x)$ denote the length of the arm of $x$, namely, $\operatorname{armlen}(x)=$ $|w u|$. A substring SAGP $y=w g u u^{R} w^{R}$ for pivot $i$ in a string $T$ is said to be a longest SAGP for pivot $i$, if for any SAGP $y^{\prime}$ for pivot $i$ in $T, \operatorname{armlen}(y) \geq \operatorname{armlen}\left(y^{\prime}\right)$.

Notice that there can be different choices of $u$ and $w$ for the longest SAGPs at the same pivot. For instance, consider string ccabcabbace. Then, $(7,1,3,2)=\mathrm{c}|\underline{\mathrm{abc}}| \mathrm{ab}|\mathrm{ba}| \mathrm{c}$ and $(7,2,3,1)=\mathrm{ca}|\underline{\mathrm{bca}}| \mathrm{b}|\mathrm{b}| \mathrm{ac}$ are both longest SAGPs (with arm length $|w u|=3$ ) for the same pivot 7, where the underlines represent the gaps. Of all longest SAGPs for each pivot $i$, we regard those that have longest palindromes $u u^{R}$ centered at $i$ as canonical longest SAGPs for pivot $i$. In the above example, $(7,1,3,2)=\mathrm{c}|\mathrm{abc}| \mathrm{ab}|\mathrm{ba}| \mathrm{c}$ is a canonical longest SAGP for pivot 7 , while $(7,2,3,1)=\mathrm{ca}|\underline{\mathrm{bca}}| \mathrm{b}|\mathrm{b}| \mathrm{ac}$ is not. Let $\operatorname{SAGP}(T)$ be the set of canonical longest SAGPs for all pivots in $T$. In this paper, we present several algorithms to compute $\operatorname{SAGP}(T)$.

A position $i$ in string $T$ is said to be of type- 1 if there exists a SAGP $w g u u^{R} w^{R}$ such that $u u^{R}$ is the maximal palindrome centered at position $i$, and is said to be of type- 2 otherwise. For instance, consider string $T=$ baaabaabaacbaabaabac of length 20. Position 13 of $T$ is of type- 1 , since there are canonical longest $\operatorname{SAGPs}(13,4,4,2)=$ abaa $|\underline{\text { baac }}|$ ba $\mid$ ab $\mid$ aaba and $(13,4,1,2)=$ abaa $|\underline{c}|$ ba $\mid$ ab $\mid$ aaba for pivot 13 , where $\mathrm{ba} \mid \mathrm{ab}$ is the maximal palindrome centered at position 13. On the other hand, Position 6 of $T$ is of type-2; the maximal palindrome centered at position 6 is aaba|abaa but there are no SAGPs in the form of $w g$ aaba|abaa $w^{R}$ for pivot 6 . The canonical longest SAGPs for pivot 6 is $(6,1,1,3)=\mathrm{a}|\underline{\mathrm{a}}| \mathrm{aba}|\mathrm{aba}| \mathrm{a}$.

For an input string $T$ of length $n$ over an integer alphabet of size $\sigma=n^{O(1)}$, we perform standard preprocessing which replaces all characters in $T$ with integers from range $[1, n]$. Namely, we radix sort the original characters in $T$, and replace each original character by its rank in the sorted order. Since the original integer alphabet is of size $n^{O(1)}$, the radix sort can be implemented with $O(1)$ number of bucket sorts, taking $O(n)$ total time. Thus, whenever we speak of a string $T$ over an integer alphabet of size $n^{O(1)}$, one can regard $T$ as a string over an integer alphabet of size $n$.

Tools: Suppose a string $T$ ends with a unique character that does not appear elsewhere in $T$. The suffix tree [23] of a string $T$, denoted by $\operatorname{STree}(T)$, is a path-compressed trie which represents all suffixes of $T$. Then, STree $(T)$ can be defined as an edge-labeled rooted tree such that (1) Every internal node is branching; (2) The out-going edges of every internal node begin with mutually distinct characters; (3) Each edge is labeled by a non-empty substring of $T$; (4) For each suffix $s$ of $T$, there is a unique leaf 
such that the path from the root to the leaf spells out $s$. It follows from the above definition of STree $(T)$ that if $n=|T|$ then the number of nodes and edges in STree $(T)$ is $O(n)$. By representing every edge label $X$ by a pair $(i, j)$ of integers such that $X=T[i . . j]$, STree $(T)$ can be represented with $O(n)$ space. For a given string $T$ of length $n$ over an integer alphabet of size $\sigma=n^{O(1)}$, STree $(T)$ can be constructed in $O(n)$ time [9]. For each node $v$ in $\operatorname{STree}(T)$, let $\operatorname{str}(v)$ denote the string spelled out from the root to $v$. According to Property (4), we sometimes identify each position $i$ in string $T$ with the leaf which represents the corresponding suffix $T[i . . n]$.

Suppose the unique character at the end of string $T$ is the lexicographically smallest in $\Sigma$. The suffix array [19] of string $T$ of length $n$, denoted $\mathrm{SA}_{T}$, is an array of size $n$ such that $\mathrm{SA}_{T}[i]=j$ iff $T[j . . n]$ is the $i$ th lexicographically smallest suffix of $T$ for $1 \leq i \leq n$. The reversed suffix array of $T$, denoted $\mathrm{SA}_{T}^{-1}$, is an array of size $n$ such that $\mathrm{SA}_{T}^{-1}\left[\mathrm{SA}_{T}[i]\right]=i$ for $1 \leq i \leq n$. The longest common prefix array of $T$, denoted $\mathrm{LCP}_{T}$, is an array of size $n$ such that $\mathrm{LCP}_{T}[1]=-1$ and $\operatorname{LCP}_{T}[i]=\operatorname{Icp}\left(T\left[\mathrm{SA}_{T}[i-1] . . n\right], T\left[\mathrm{SA}_{T}[i] . . n\right]\right)$ for $2 \leq i \leq n$. The arrays $\mathrm{SA}_{T}, \mathrm{SA}_{T}^{-1}$, and $\mathrm{LCP}_{T}$ for a given string $T$ of length $n$ over an integer alphabet of size $\sigma=n^{O(1)}$ can be constructed in $O(n)$ time [15, 16].

For a rooted tree $\mathcal{T}$, the lowest common ancestor $\operatorname{LCA}_{\mathcal{T}}(u, v)$ of two nodes $u$ and $v$ in $\mathcal{T}$ is the deepest node in $\mathcal{T}$ which has $u$ and $v$ as its descendants. It is known that after a linear-time preprocessing on the input tree, querying $\operatorname{LCA}_{\mathcal{T}}(u, v)$ for any two nodes $u, v$ can be answered in constant time [2].

Consider a rooted tree $\mathcal{T}$ where each node is either marked or unmarked. For any node $v$ in $\mathcal{T}$, let $\operatorname{NMA}_{\mathcal{T}}(v)$ denote the deepest marked ancestor of $v$. There exists a linear-space algorithm which marks any unmarked node and returns $\operatorname{NMA}_{\mathcal{T}}(v)$ for any node $v$ in amortized $O(1)$ time [24].

Let $A$ be an integer array of size $n$. A range minimum query $\operatorname{RMQ}_{A}(i, j)$ of a given pair $(i, j)$ of indices $(1 \leq i \leq j \leq n)$ asks an index $k$ in range $[i, j]$ which stores the minimum value in $A[i . . j]$. After $O(n)$-time preprocessing on $A, \mathrm{RMQ}_{A}(i, j)$ can be answered in $O(1)$ time for any given pair $(i, j)$ of indices [2].

Let $S$ be a set of $m$ integers from universe $[1, n]$, where $n$ fits in a single machine word. A predecessor (resp. successor) query for a given integer $x$ to $S$ asks the largest (resp. smallest) value in $S$ that is smaller (resp. larger) than $x$. Let $u(m, n), q(m, n)$ and $s(m, n)$ denote the time for updates (insertion/deletion) of elements, the time for predecessor/successor queries, and the space of a dynamic predecessor/successor data structure. Using a standard balanced binary search tree, we have $u(m, n)=q(m, n)=O(\log m)$ time and $s(n, m)=O(m)$ space. The Y-fast trie [25] achieves $u(m, n)=q(m, n)=O(\log \log m)$ expected time and $s(n, m)=O(m)$ space, while the van Emde Boas tree [22] does $u(m, n)=q(m, n)=O(\log \log m)$ worst-case time and $s(n, m)=O(n)$ space.

\section{Algorithms for computing canonical longest SAGPs}

In this section, we present several algorithms to compute the set $\operatorname{SAGP}(T)$ of canonical longest $\operatorname{SAGPs}$ for all pivots in a given string $T$.

Let $\operatorname{Pos}_{1}(T)$ and $\operatorname{Pos}_{2}(T)$ be the sets of type- 1 and type- 2 positions in $T$, respectively. Let $\operatorname{SAGP}(T, i)$ be the subset of SAGP $(T)$ whose elements are canonical longest SAGPs for pivot $i$. Let $\operatorname{SAGP}_{1}(T)=$ $\bigcup_{i \in \operatorname{Pos}_{1}(T)} \operatorname{SAGP}(T, i)$ and $\operatorname{SAGP}_{2}(T)=\bigcup_{i \in \operatorname{Pos}_{2}(T)} \operatorname{SAGP}(T, i)$. Clearly $\operatorname{SAGP}_{1}(T) \cup \operatorname{SAGP}_{2}(T)=\operatorname{SAGP}(T)$ and $\mathrm{SAGP}_{1}(T) \cap \mathrm{SAGP}_{2}(T)=\emptyset$. The following lemma gives an useful property to characterize the type-1 positions of $T$.

Lemma 1. Let $i$ be any type-1 position of a string $T$ of length $n$. Then, a SAGP wguu ${ }^{R} w^{R}$ is a canonical longest $S A G P$ for pivot $i$ iff $u u^{R}$ is the maximal palindrome centered at $i$ and $w^{R}$ is the longest non-empty prefix of $T\left[i+\left|u^{R}\right|+1 . . n\right]$ such that $w$ occurs at least once in $T[1 . . i-|u|-1]$.

Proof. $(\Rightarrow)$ Assume on the contrary that $u u^{R}$ is not the maximal palindrome centered at $i$, and let $x u u^{R} x^{R}$ be the maximal palindrome centered at position $i$ with $|x| \geq 1$. If $w^{R}=x^{R}$, then since position 


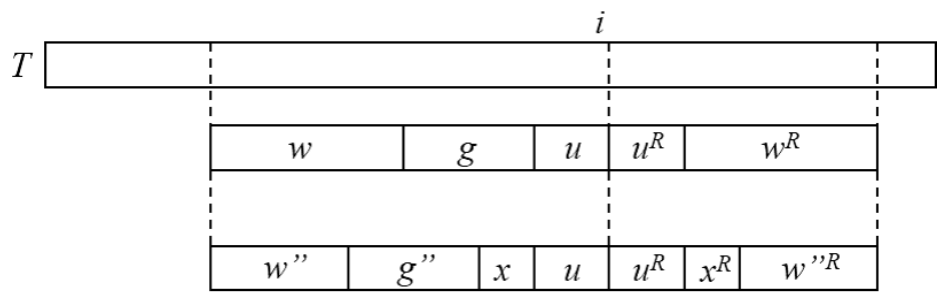

Figure 1: Illustration for a necessary condition for a canonical longest SAGP (proof of $(\Rightarrow)$ for Lemma 1): $w g u u^{R} w^{R}$ is a canonical longest SAGP for pivot $i$. For the same pivot $i$, there cannot exist a SAGP $w^{\prime \prime} g^{\prime \prime} x u u^{R} x^{R} w^{\prime \prime R}$ where $x u u^{R} x^{R}$ is the maximal palindrome centered at $i$ and $w^{\prime \prime}$ is a prefix of $w$, since it contradicts that $w g u u^{R} w^{R}$ is a canonical longest SAGP for $i$.

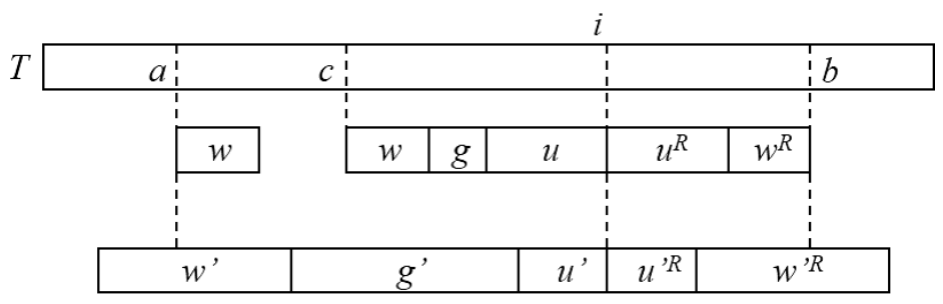

Figure 2: Illustration for a sufficient condition for a canonical longest SAGP (proof of $(\Leftarrow)$ for Lemma 1): $u u^{R}$ is the maximal palindrome centered at $i$ and $w^{R}$ is the longest prefix of $T\left[i+\left|u^{R} w^{R}\right|+1 . . n\right]$ such that $w$ occurs at least once in $T[1 . . i-|u|-1]$, and thus $c \neq b$. Then, there cannot exist a longer SAGP $w^{\prime} g^{\prime} u^{\prime} u^{\prime R} w^{\prime R}$ for the same pivot $i$, since $a \neq b$.

$i$ is of type-1, there must be a SAGP $w^{\prime} g^{\prime} x u u^{R} x^{R} w^{\prime R}$ with $\left|w^{\prime}\right| \geq 1$ for pivot $i$, but this contradicts that $w g u u^{R} w^{R}$ is a longest SAGP for pivot $i$. Hence $x^{R}$ is a proper prefix of $w^{R}$. See Figure 1. Let $x^{R} w^{\prime \prime R}=w^{R}$. Since $w^{\prime \prime R}$ is a non-empty suffix of $w^{R}, w^{\prime \prime}$ is a non-empty prefix of $w$. This implies that there exists a SAGP $w^{\prime \prime} g^{\prime \prime} x u u^{R} x^{R} w^{\prime \prime R}$ for pivot $i$. However, this contradicts that $w g u u^{R} w^{R}$ is a canonical longest SAGP for pivot $i$. Consequently, $u u^{R}$ is the maximal palindrome centered at $i$, and now it immediately follows that $w^{R}$ is the longest non-empty prefix of $T\left[i+\left|u^{R}\right|+1 . . n\right]$ such that $w$ occurs at least once in $T[1 . . i-|u|-1]$.

$(\Leftarrow)$ First, we show that $w g u u^{R} w^{R}$ is a longest SAGP for pivot $i$. See Figure 2, Let $u^{\prime}$ be any proper suffix of $u$, and assume on the contrary that there exists a SAGP $w^{\prime} g^{\prime} u^{\prime} u^{\prime} w^{\prime R}$ for pivot $i$ such that $\left|w^{\prime} u^{\prime}\right|>|w u|$. Since $\left|u^{\prime}\right|<|u|$, the occurrence of $w^{R}$ at position $i+\left|u^{R}\right|$ is completely contained in the occurrence of $w^{\prime R}$ at position $i+\left|u^{\prime R}\right|$. This implies that any occurrence of $w^{\prime}$ to the left of $u^{\prime} u^{\prime R}$ completely contains an occurrence of $w$, reflected from the occurrence of $w^{R}$ in $w^{\prime R}$. However, the character $a$ that immediately precedes the occurrence of $w$ in $w^{\prime}$ must be distinct from the character $b$ that immediately follows $w^{R}$, namely $a \neq b$. This contradicts that $w^{\prime} g^{\prime} u^{\prime} u^{\prime R} w^{\prime R}$ is a SAGP for pivot $i$. Hence, $w g u u^{R} w^{R}$ is a longest SAGP for pivot $i$. Since $u u^{R}$ is the maximal palindrome centered at $i$, we cannot extend $u$ to its left nor $u^{R}$ to its right for the same center $i$. Thus, $w g u u^{R} w^{R}$ is a canonical longest SAGP for pivot $i$.

We define two arrays Pals and LMost as follows:

$$
\begin{aligned}
\operatorname{Pals}[i] & =\{r \mid T[i-r+1 . . i+r] \text { is a maximal palindrome in } T \text { for pivot } i\} . \\
\operatorname{LMost}[c] & =\min \{i \mid T[i]=c\} \text { for } c \in \Sigma .
\end{aligned}
$$




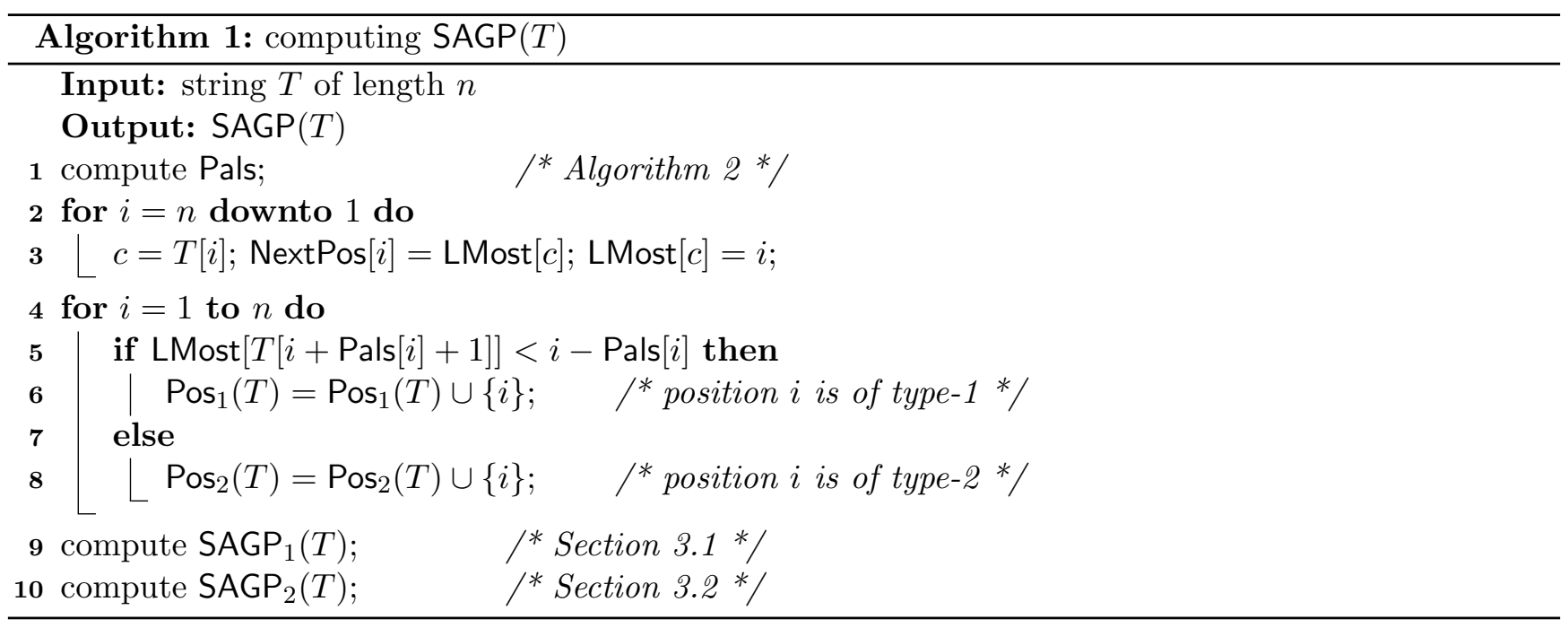

By Lemma 1, a position $i$ in $T$ is of type-1 iff $\operatorname{LMost}[T[i+\operatorname{Pals}[i]+1]]<i-\operatorname{Pals}[i]$.

Lemma 2. Given a string $T$ of length $n$ over an integer alphabet of size $n^{O(1)}$, we can determine whether each position $i$ of $T$ is of type-1 or type-2 in a total of $O(n)$ time and space.

Proof. Let $u u^{R}$ be the maximal palindrome centered at $i$. Observe that, by Lemma 1, $i$ is a type-1 position iff the character $a=T\left[i+\left|u^{R}\right|+1\right]$ which immediately follows $u^{R}$ occurs in $T[1 . . i-|u|-1]$. Let $\Sigma_{T}$ be the set of distinct characters occurring in $T$. We construct an array LMost of size $\left|\Sigma_{T}\right|$ such that for each $1 \leq j \leq\left|\Sigma_{T}\right|$, LMost $[j]$ stores the leftmost occurrence of the lexicographically $j$ th character in $T$. Using the above observation and the array LMost, we can determine in $O(1)$ time whether a given position $i$ of $T$ is of type- 1 or type- 2 by LMost $[T[i+\operatorname{Pals}[i]+1]]<i-\operatorname{Pals}[i]$. We can sort the characters in $\Sigma_{T}$ in $O(n)$ by constructing $\mathrm{SA}_{T}$ in $O(n)$ time and space.

By Lemma 1 and Lemma 2, we can consider an algorithm to compute $\operatorname{SAGP}(T)$ by computing $\mathrm{SAGP}_{1}(T)$ and $\mathrm{SAGP}_{2}(T)$ separately, as shown in Algorithm 1. In this algorithm, we also construct an auxiliary array NextPos defined by $\operatorname{NextPos}[i]=\min \{j \mid i<j, T[i]=T[j]\}$ for each $1 \leq i \leq n$, which will be used in Section 3.2 .

Lemma 3. Algorithm 1 correctly computes $\operatorname{SAGP}(T)$.

Proof. In line 1, we firstly compute an array Pals. Pals $[i]$ stores radius $r$ of maximal palindrome centered at $i$. We can compute Pals in $O(n)$ time and space applying Manacher's algorithm [18]. We show how to compute Pals in Algorithm 2, In the first for-loop, we construct auxiliary arrays LMost and NextPos. The correctness of the computation of these arrays is obvious. We use NextPos when computing SAGP . In line 6, since we correctly determine which each position of $T$ is of type-1 or type-2 by Lemma 2 , we must compute $\operatorname{Pos}_{1}(T)$ and $\operatorname{Pos}_{2}(T)$ in the second for-loop. Therefore, by referring each element of $\operatorname{Pos}_{1}(T)$ and $\operatorname{Pos}_{2}(T)$ respectively, we can compute $\operatorname{SAGP}_{1}(T)$ and $\operatorname{SAGP}_{2}(T)$, namely $\operatorname{SAGP}(T)$.

In the following subsections, we present algorithms to compute $\operatorname{SAGP}_{1}(T)$ and $\mathrm{SAGP}_{2}(T)$ respectively, assuming that the arrays Pals, LMost and NextPos have already been computed. 


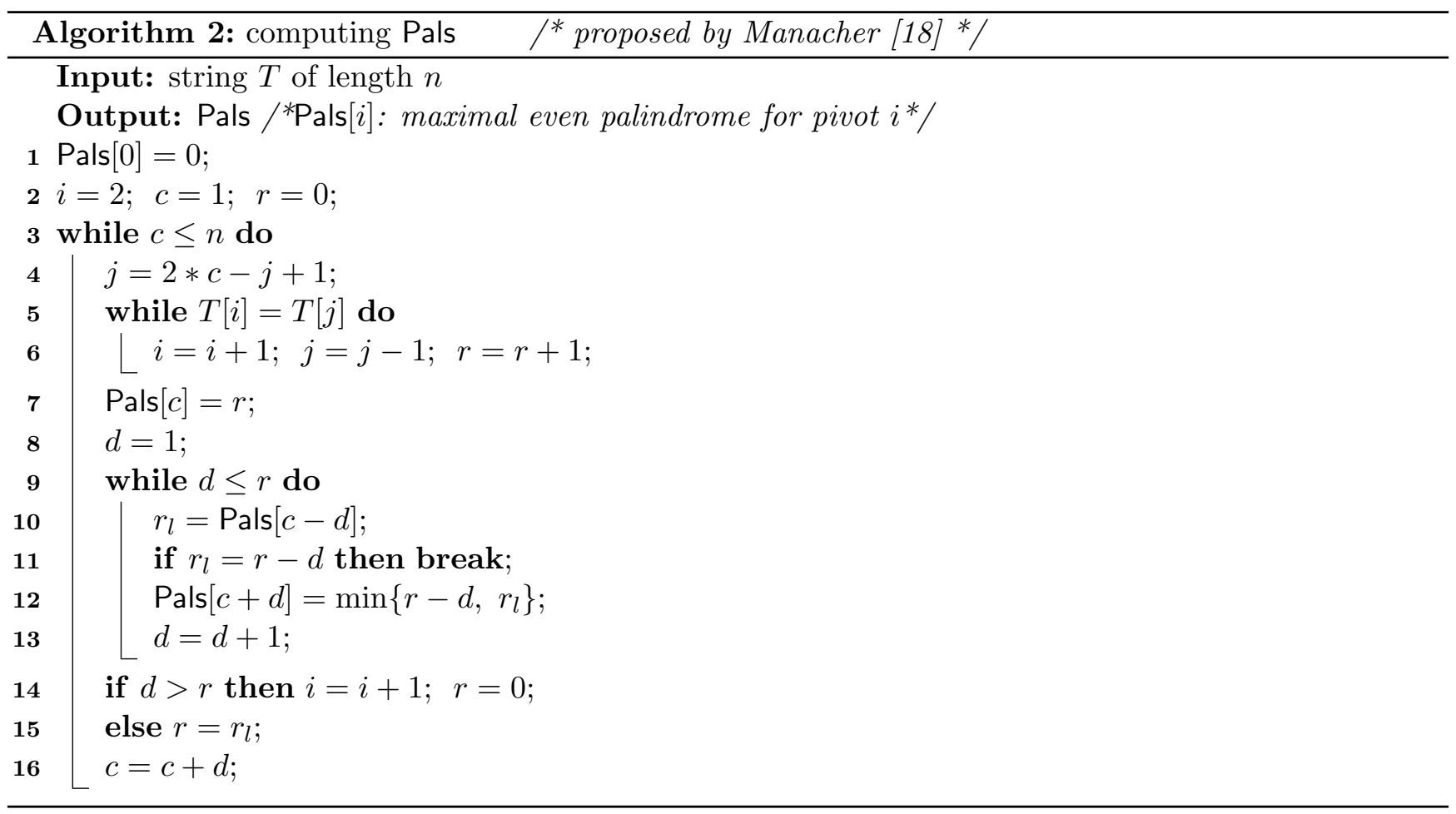

\subsection{Computing $\operatorname{SAGP}_{1}(T)$ for type-1 positions}

In what follows, we present several algorithms corresponding to the line 9 in Algorithm 1 . Lemma 1 . allows us greedy strategies to compute the longest prefix $w^{R}$ of $T[i+\operatorname{Pals}[i]+1 . . n]$ such that $w$ occurs in $T[1 . . i-\operatorname{Pals}[i]-1]$.

\subsubsection{Naïve quadratic-time algorithm with RMQs}

Let $T^{\prime}=T \$ T^{R} \#$. We construct the suffix array $\mathrm{SA}_{T^{\prime}}$, the reversed suffix array $\mathrm{SA}_{T^{\prime}}^{-1}$, and the LCP array $\mathrm{LCP}_{T^{\prime}}$ for $T^{\prime}$.

For each Pals $[i]$ in $T$, for each gap size $G=1, \ldots, i-\operatorname{Pals}[i]-1$, we compute $W=\operatorname{lcp}(T[1 . . i-$ Pals $[i]-$

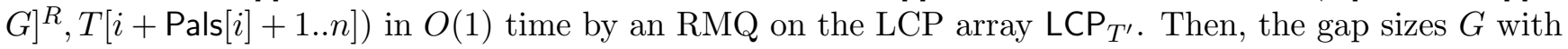
largest values of $W$ give all longest SAGPs for pivot $i$. Since we test $O(n)$ gap sizes for every pivot $i$, it takes a total of $O\left(n^{2}\right)$ time to compute $\operatorname{SAGP}_{1}(T)$. The working space of this method is $O(n)$.

\subsubsection{Simple quadratic-time algorithm based on suffix array}

Given a string $T$, we construct $\mathrm{SA}_{T^{\prime}}, \mathrm{SA}_{T^{\prime}}^{-1}$, and $\mathrm{LCP}_{T^{\prime}}$ for string $T^{\prime}=T \$ T^{R} \#$ as in the previous subsection. Further, for each position $n+2 \leq j \leq 2 n+1$ in the reversed part $T^{R}$ of $T^{\prime}=T \$ T^{R} \#$, let $\mathrm{op}(j)$ denote its "original" position in the string $T$, namely, let op $(j)=2 n-j+2$. Let $e$ be any entry of $\mathrm{SA}_{T^{\prime}}$ such that $\mathrm{SA}_{T^{\prime}}[e] \geq n+2$. We associate each such entry of $\mathrm{SA}_{T^{\prime}}$ with op $\left(\mathrm{SA}_{T^{\prime}}[e]\right)$.

Let $\mathrm{SA}_{T^{\prime}}[k]=i+\operatorname{Pals}[i]+1$, namely, the $k$-th entry of $\mathrm{SA}_{T^{\prime}}$ corresponds to the suffix $T[i+\operatorname{Pals}[i]+1 . . n]$ of $T$. Now, the task is to find the longest prefix $w^{R}$ of $T[i+\operatorname{Pals}[i]+1 . . n]$ such that $w$ occurs completely inside $T[1 . . i-\operatorname{Pals}[i]-1]$. Let $b=i-\operatorname{Pals}[i]+1$, namely, $b$ is the beginning position of the maximal palindrome $u u^{R}$ centered at $i$. We can find $w$ for any maximal SAGP $w g u u^{R} w^{R}$ for pivot $i$ by traversing $\mathrm{SA}_{T^{\prime}}$ from the $k$-th entry forward and backward, until we encounter the nearest entries $p<k$ and $q>k$ 
on $\mathrm{SA}_{T^{\prime}}$ such that op $\left(\mathrm{SA}_{T^{\prime}}[p]\right)<b-1$ and op $\left(\mathrm{SA}_{T^{\prime}}[q]\right)<b-1$, if they exist. The size $W$ of $w$ is equal to

$$
\max \left\{\min \left\{\mathrm{LCP}_{T^{\prime}}[p+1], \ldots, \mathrm{LCP}_{T^{\prime}}[k]\right\}, \min \left\{\mathrm{LCP}_{T^{\prime}}[k+1], \ldots, \mathrm{LCP}_{T^{\prime}}[q]\right\}\right\} .
$$

Assume w.l.o.g. that $p$ gives a larger lcp value with $k$, i.e. $W=\min \left\{\mathrm{LCP}_{T^{\prime}}[p+1], \ldots, \mathrm{LCP}_{T^{\prime}}[k]\right\}$. Let $s$ be the largest entry of $\mathrm{SA}_{T^{\prime}}$ such that $s<p$ and $\mathrm{LCP}_{T^{\prime}}[s+1]<W$. Then, any entry $t$ of $\mathrm{SA}_{T^{\prime}}$ such that $s<t \leq p$ and op $\left(\mathrm{SA}_{T^{\prime}}[t]\right)<b-1$ corresponds to an occurrence of a longest SAGP for pivot $i$, with gap size $b-\mathrm{op}\left(\mathrm{SA}_{T^{\prime}}[t]\right)-1$. We output longest $\operatorname{SAGP}\left(i, W, b-\mathrm{op}\left(\mathrm{SA}_{T^{\prime}}[t]\right)-1,|u|\right)$ for each such $t$. The case where $q$ gives a larger lcp value with $k$, or $p$ and $q$ give the same lcp values with $k$ can be treated similarly.

We find $p$ and $s$ by simply traversing $\mathrm{SA}_{T^{\prime}}$ from $k$. Since the distance from $k$ to $s$ is $O(n)$, the above algorithm takes $O\left(n^{2}\right)$ time. The working space is $O(n)$.

\subsubsection{Algorithm based on suffix array and predecessor/successor queries}

Let occ $_{1}=\left|\mathrm{SAGP}_{1}(T)\right|$. For any position $r$ in $T$, we say that the entry $j$ of $\mathrm{SA}_{T^{\prime}}$ is active w.r.t. $r$ iff $\mathrm{op}\left(\mathrm{SA}_{T^{\prime}}[j]\right)<r-1$. Let Active $(r)$ denote the set of active entries of $\mathrm{SA}_{T^{\prime}}$ for position $r$, namely, Active $(r)=\left\{j \mid\right.$ op $\left.\left(\mathrm{SA}_{T^{\prime}}[j]\right)<r-1\right\}$.

Let $t_{1}=p$, and let $t_{2}, \ldots, t_{h}$ be the decreasing sequence of entries of $\mathrm{SA}_{T^{\prime}}$ which correspond to the occurrences of longest SAGPs for pivot $i$. Notice that for all $1 \leq \ell \leq h$ we have op $\left(\mathrm{SA}_{T^{\prime}}\left[t_{\ell}\right]\right)<b-1$ and hence $t_{\ell} \in \operatorname{Active}(b)$, where $b=i-|u|+1$. Then, finding $t_{1}$ reduces to a predecessor query for $k$ in $\operatorname{Active}(b)$. Also, finding $t_{\ell}$ for $2 \leq \ell \leq h$ reduces to a predecessor query for $t_{\ell-1}$ in $\operatorname{Active}(b)$.

To effectively use the above observation, we compute an array $U$ of size $n$ from Pals such that $U[b]$ stores a list of all maximal palindromes in $T$ which begin at position $b$ if they exist, and $U[b]$ is nil otherwise. $U$ can be computed in $O(n)$ time e.g., by bucket sort. After computing $U$, we process $b=1, \ldots, n$ in increasing order. Assume that when we process a certain value of $b$, we have maintained a dynamic predecessor/successor query data structure for Active $(b)$. The key is that the same set Active $(b)$ can be used to compute the longest SAGPs for every element in $U[b]$, and hence we can use the same predecessor/successor data structure for all of them. After processing all elements in $U[b]$, we insert all elements of Active $(b+1) \backslash \operatorname{Active}(b)$ to the predecessor/successor data structure. Each element to insert can be easily found in constant time.

Since we perform $O\left(n+o c c_{1}\right)$ predecessor/successor queries and $O(n)$ insertion operations in total, we obtain the following theorem.

Theorem 1. Given a string $T$ of size $n$ over an integer alphabet of size $\sigma=n^{O(1)}$, we can compute $\mathrm{SAGP}_{1}(T)$ in $O\left(n(u(n, n)+q(n, n))+\right.$ occ $\left.c_{1} \cdot q(n, n)\right)$ time with $O(n+s(n, n))$ space by using the suffix array and a predecessor/successor data structure, where occ ${ }_{1}=\left|\operatorname{SAGP}_{1}(T)\right|$.

Since every element of Active $(b)$ for any $b$ is in range $[1,2 n+2]$, we can employ the van Emde Boas tree [22] as the dynamic predecessor/successor data structure using $O(n)$ total space. Thus we obtain the following theorem.

Theorem 2. Given a string $T$ of size $n$ over an integer alphabet of size $\sigma=n^{O(1)}$, we can compute $\mathrm{SAGP}_{1}(T)$ in $O\left(\left(n+o c c_{1}\right) \log \log n\right)$ time and $O(n)$ space by using the suffix array and the van Emde Boas tree, where occ $1=\left|\mathrm{SAGP}_{1}(T)\right|$.

Example 1. Let $T=$ acacabaabca and $T^{\prime}=$ acacabaabca $\$ a c b a a b a c a c a \#$, where $T^{\prime}=T \$ T^{R} \#$. First, we compute Pals and the array $U$. Assume we are now processing position $b=6$ in $T$, then $U[6]=\{(6,9)\}$, where $(6,9)$ represents the maximal palindrome $T[6 . .9]=$ baab. Thus we consider pivot $i=b+\lceil(9-6+$ 1) $/ 2\rceil-1=7$. 


\begin{tabular}{|c|c|c|c|c|c|c|c|c|c|c|c|c|c|c|c|c|c|c|c|c|c|}
\hline & 2 & 23 & 4 & 5 & 6 & $C$ & & & & & & & & & & & & & & & \\
\hline$S \mathrm{~A}_{T^{\prime}}$ & \begin{tabular}{|l|l|}
12 & 24
\end{tabular} & 11 & 23 & \begin{tabular}{|l|}
16 \\
\end{tabular} & 7 & \begin{tabular}{l|l}
5 & 1
\end{tabular} & & \begin{tabular}{l|l|l}
8 & 21
\end{tabular} & 3 & 19 & 1 & \begin{tabular}{l|l}
13 & 1
\end{tabular} & & & & & & & 20 & 2 & 14 \\
\hline $\mathrm{LCP}_{T^{\prime}}$ & \begin{tabular}{|l|l}
-1 & 0
\end{tabular} & \begin{tabular}{|l|l|}
0 & 0
\end{tabular} & 1 & 1 & 3 & 13 & 3 & \begin{tabular}{l|l}
2 & 1
\end{tabular} & 3 & 3 & 5 & 2 & 0 & 42 & 2 & 1 & 0 & 2 & 2 & 4 & 1 \\
\hline $\mathrm{SA}_{T^{\prime}}^{-1}$ & \begin{tabular}{|l|l|l|l|}
13 & 23 \\
\end{tabular} & 3 & 21 & 7 & 16 & \begin{tabular}{l|l}
6 & 9 \\
\end{tabular} & 9 & \begin{tabular}{l|l}
8 & 19 \\
\end{tabular} & 93 & 1 & 14 & & & 5 & & & & & 20 & 4 & 2 \\
\hline
\end{tabular}

Figure 3: $\mathrm{SA}_{T^{\prime}}, \mathrm{LCP}_{T^{\prime}}$ and $\mathrm{SA}_{T^{\prime}}^{-1}$ for string $T^{\prime}=$ acacabaabca $\$$ acbaabacaca\#.

First, we construct the suffix array $\mathrm{SA}_{T^{\prime}}$, the reversed suffix array $\mathrm{SA}_{T^{\prime}}^{-1}$, and the LCP array $\mathrm{LCP}_{T^{\prime}}$ for $T^{\prime}$. Figure 3 shows these arrays. Let $k$ be the integer such that $\mathrm{SA}_{T^{\prime}}[k]=i+\lceil(9-6+1) / 2\rceil+1=7+3=10$, namely $k=19$. This can be obtained from $\mathrm{SA}^{-1}[10]=19$ (see Figure 3). To compute the longest $w$, we traverse $\mathrm{SA}_{T^{\prime}}$ [19] forward and backward, until we encounter the nearest entries $p<k$ and $q>k$ on $\mathrm{SA}_{T^{\prime}}$ such that $\mathrm{op}\left(\mathrm{SA}_{T^{\prime}}[p]\right)<5$ and $\mathrm{op}\left(\mathrm{SA}_{T^{\prime}}[q]\right)<5$. Note that these are equivalent to predecessor $/$ successor queries for 19, respectively. Then, we can find $p=10$ and $q=20$. Then, the size $W$ of $w$ is computed by

$$
W=\max \left\{\min \left\{\mathrm{LCP}_{T^{\prime}}[11], \ldots, \mathrm{LCP}_{T^{\prime}}[19]\right\}, \min \left\{\mathrm{LCP}_{T^{\prime}}[20], \ldots, \mathrm{LCP}_{T^{\prime}}[20]\right\}\right\}
$$

and we obtain $W=2$. In this case, $q=20$ gives a larger lcp value with $k=19$. Thus, we output a canonical longest $\operatorname{SAGP}(7,2,3,2)=\mathrm{ac} \mid$ aca $|\mathrm{ba}| \mathrm{ab} \mid \mathrm{ca}$. We further traverse $\mathrm{SA}_{T^{\prime}}$ from the 20 th entry backward as long as successive entries $s$ fulfill $\mathrm{LCP}_{T^{\prime}}[s+1] \geq W$. Then, we find $s=22$, thus we output a canonical longest $\operatorname{SAGP}(7,2,1,2)=\mathrm{ac}|\underline{\mathrm{a}}| \mathrm{ba}|\mathrm{ab}| \mathrm{ca}$. We further traverse $\mathrm{SA}_{T^{\prime}}$ from the 17 th entry backward, finally we reach the 24th entry of $\mathrm{SA}_{T^{\prime}}$, which is the last entry of the suffix array. Therefore, we finish the process for position $b=6$.

\subsubsection{Optimal-time algorithm based on suffix tree}

In this subsection, we show that the problem can be solved in optimal time and space, using the following three suffix trees regarding the input string $T$. Let $\mathcal{T}_{1}=\operatorname{STree}\left(T \$ T^{R} \#\right)$ for string $T \$ T^{R} \#$ of length $2 n+2$, and $\mathcal{T}_{2}=\operatorname{STree}\left(T^{R} \#\right)$ of length $n+1$. These suffix trees $\mathcal{T}_{1}$ and $\mathcal{T}_{2}$ are static, and thus can be constructed offline, in $O(n)$ time for an integer alphabet. We also maintain a growing suffix tree $\left.\mathcal{T}_{2}^{\prime}=\operatorname{STree}\left(T^{R}[k . . n]\right) \#\right)$ for decreasing $k=n, \ldots, 1$.

Lemma 4. Given $\mathcal{T}_{2}=\operatorname{STree}\left(T^{R} \#\right)$, we can maintain $\mathcal{T}_{2}^{\prime}=\operatorname{STree}\left(T^{R}[k . . n] \#\right)$ for decreasing $k=n, \ldots, 1$ incrementally, in $O(n)$ total time for an integer alphabet of size $n^{O(1)}$.

Proof. We build an LCA data structure on $\mathcal{T}_{2}=\operatorname{STree}\left(T^{R} \#\right)$. We also construct a level ancestor data structure [3] on $\mathcal{T}_{2}$ in linear preprocessing time and space, so that later, given a node $u$ and a positive integer $\ell$, the $\ell$ th ancestor of $u$ in $\mathcal{T}_{2}$ can be answered in $O(1)$ time. Additionally, we use $\mathrm{SA}_{T^{R} \#}$ and $\mathrm{SA}_{T^{R} \#}^{-1}$ in our algorithm, and throughout this proof we abbreviate $\mathrm{SA}_{T^{R} \#}$ as $\mathrm{SA}$ and $\mathrm{SA}_{T^{R}}^{-1}$ as $\mathrm{SA}^{-1}$ for simplicity. Let PLV and NLV be arrays of size $n+1$ each, such that for every $1 \leq j \leq n+1$,

$$
\begin{aligned}
& \operatorname{PLV}[j]=\max \left(\left\{j^{\prime} \mid 1 \leq j^{\prime}<j, \mathrm{SA}\left[j^{\prime}\right]>\operatorname{SA}[j]\right\} \cup\{-\infty\}\right), \\
& \operatorname{NLV}[j]=\min \left(\left\{j^{\prime} \mid j<j^{\prime} \leq n+1, \mathrm{SA}\left[j^{\prime}\right]>\operatorname{SA}[j]\right\} \cup\{\infty\}\right)
\end{aligned}
$$

Intuitively, PLV $[j]$ and NLV $[j]$ indicate the entries of SA that correspond to the lexicographically closest suffixes to the left and to the right of the suffix $T^{R}[\mathrm{SA}[j] . . n] \#$ which occur positions larger than $j$, respectively. If such entries do not exist, then let $\operatorname{PLV}[j]=-\infty$ and $\operatorname{NLV}[j]=\infty$. See also Figure 4 for concrete examples of PLV and NLV arrays. 


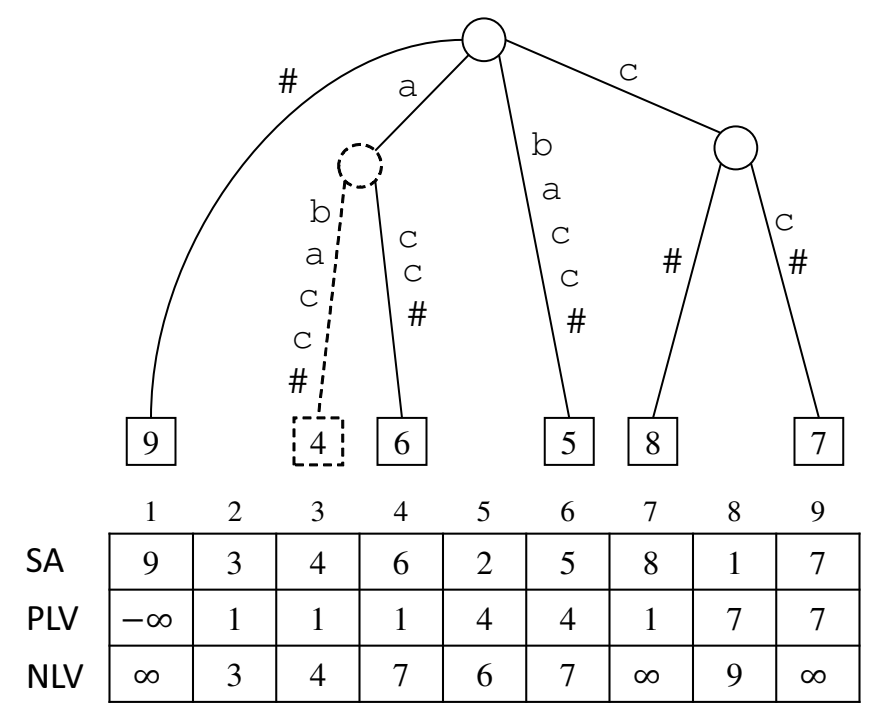

Figure 4: Illustration for the proof of Lemma 4. Consider string $T=$ ccabaabc. Here, we illustrate incremental construction of the growing suffix tree $\mathcal{T}_{2}^{\prime}$ for its reversed string $T^{R} \#=$ cbaabacc\#. Assume we have constructed $\mathcal{T}_{2}^{\prime}=\operatorname{STree}\left(T^{R}[5 . .8] \#\right)=\mathrm{S} \operatorname{Tree}($ bacc\#), and we are inserting a new leaf for the next suffix $T^{R}[4 . .8] \#=$ abacc\# starting at position 4 in $T^{R} \#$. $\mathrm{SA}^{-1}[4]=3$, hence we focus on the suffixes of $T^{R} \#$ starting at positions $\mathrm{SA}[\mathrm{PLV}[3]]=\mathrm{SA}[1]=9$ and $\mathrm{SA}[\mathrm{NLV}[3]]=\mathrm{SA}[4]=6$. Since $\operatorname{Icp}\left(T^{R}[9 . .8] \#, T^{R}[4 . .8] \#\right)=\operatorname{Icp}(\#, \operatorname{abacc} \#)=0<\operatorname{Icp}\left(T^{R}[4 . .8] \#, T^{R}[6 . .8] \#\right)=\operatorname{Icp}(\operatorname{abacc} \#, \operatorname{acc} \#)=1$, we split the corresponding edge and insert the new leaf SA[3] $=4$ as the left neighbor of the leaf 6 .

Suppose we have constructed $\mathcal{T}_{2}^{\prime}=\operatorname{STree}\left(T^{R}[k+1 . . n] \#\right)$ up to position $k+1$, and we wish to update it with the new character $T^{R}[k]$ at position $k$. We maintain an NMA data structure on the full suffix tree $\mathcal{T}_{2}$ such that a node in $\mathcal{T}_{2}$ is marked iff it exists in the growing suffix tree $\mathcal{T}_{2}^{\prime}$. Namely, in each step of our algorithm, the induced tree with the marked nodes of $\mathcal{T}_{2}$ coincides with $\mathcal{T}_{2}^{\prime}$.

Now, what is required here is to insert a new leaf corresponding to the suffix $T^{R}[k . . n] \#$ to the growing suffix tree. If we use a variant of Weiner's algorithm [23, we can do this in $O(\log \sigma)$ (amortized) time, but this becomes $O(\log n)$ for integer alphabets of size $\sigma=n^{O(1)}$, and thus it is not enough for our goal. To achieve $O(1)$ update time per character, we utilize the power of the full suffix tree $\mathcal{T}_{2}=\mathrm{STree}\left(T^{R} \#\right)$ and four arrays SA, SA ${ }^{-1}$, PLV, and NLV.

In what follows, we focus on the case where $\operatorname{PLV}\left[\mathrm{SA}^{-1}[k]\right] \neq-\infty$ and $\operatorname{NLV}\left[\mathrm{SA}^{-1}[k]\right] \neq \infty$. The case where $\operatorname{PLV}\left[\mathrm{SA}^{-1}[k]\right]=\infty$ or $\operatorname{NLV}\left[\mathrm{SA}^{-1}[k]\right]=\infty$ is simpler and can be treated similarly. A key observation is that there is a one-to-one correspondence between every leaf of $\operatorname{STree}\left(T^{R}[k+1 . . n] \#\right)$ and an entry of SA which stores a position in $T^{R} \#$ which is larger than $k$. Hence, SA $\left[\mathrm{PLV}\left[\mathrm{SA}^{-1}[k]\right]\right]$ and SA $\left[\mathrm{NLV}\left[\mathrm{SA}^{-1}[k]\right]\right]$ will be, respectively, the left and right neighboring leaves of the new leaf $k$ in the updated suffix tree $\operatorname{STree}\left(T^{R}[k . . n] \#\right)$.

Given the new position $k$, we compute the following values $L$ and $R$ :

$$
\begin{aligned}
L & =\operatorname{lcp}\left(\left(T^{R} \#\right)\left[\operatorname{SA}\left[\operatorname{PLV}\left[\operatorname{SA}^{-1}[k]\right] . . n+1\right]\right],\left(T^{R} \#\right)[k . . n+1]\right), \\
R & =\operatorname{lcp}\left(\left(T^{R} \#\right)[k . . n+1],\left(T^{R} \#\right)\left[\operatorname{SA}\left[\operatorname{NLV}\left[\operatorname{SA}^{-1}[k]\right] . . n+1\right]\right]\right) .
\end{aligned}
$$

Let $v_{L}$ and $v_{R}$ be the LCA nodes in the full suffix tree $\mathcal{T}_{2}$ that correspond to the LCP values $L$ and $R$, respectively (i.e., the string depths of $v_{L}$ and $v_{R}$ are respectively $L$ and $R$ ). Both $v_{L}$ and $v_{R}$ can be found in $O(1)$ time by LCA queries on the full suffix tree $\mathcal{T}_{2}$. Depending on the values of $L$ and $R$, we have the following cases. 
- If $L \geq R$, then leaf SA[PLV[SA $\left.\left.{ }^{-1}[k]\right]\right]$ will be the left neighbor of the new leaf $k$ in the updated suffix tree. We then find the parent parent $\left(v_{L}\right)$ of $v_{L}$ in the growing tree $\mathcal{T}_{2}^{\prime}$, by an NMA query from $v_{L}$ on the full suffix tree $\mathcal{T}_{2}$. Then, in the growing tree, we split the corresponding out-going edge of parent $\left(v_{L}\right)$ with the string depth of $v_{L}$ if necessary, and insert a new leaf $k$. In case where the parent of this leaf $k$ was newly created after splitting the edge, then we mark the corresponding node in the full suffix tree $\mathcal{T}_{2}$.

What remains is how to efficiently locate this corresponding out-going edge of parent $\left(v_{L}\right)$ to be split. For this sake, we associate each edge $f$ of $\mathcal{T}_{2}^{\prime}$ with the first edge of the path in $\mathcal{T}_{2}$ that corresponds to $f$. We also precompute the depths of all nodes in the full suffix tree $\mathcal{T}_{2}$ by a standard linear-time tree traversal. Let $u$ be the (marked) child of parent $\left(v_{L}\right)$ in the growing tree $\mathcal{T}_{2}^{\prime}$ such that the edge from parent $\left(v_{L}\right)$ to $u$ is split and $v_{L}$ is newly inserted in between. Let $x$ be the label of the edge from parent $\left(v_{L}\right)$ to $u$ in $\mathcal{T}_{2}^{\prime}$, and let $e$ be the first edge of the corresponding path of $\mathcal{T}_{2}$ that spells out $x$. Since we know the depth of parent $\left(v_{L}\right)$ in the full suffix tree $\mathcal{T}_{2}$, we can find $e$ in $O(1)$ time by a level ancestor query from leaf SA PLV $\left.\left[\mathrm{SA}^{-1}[k]\right]\right]$ on the full suffix tree $\mathcal{T}_{2}$. Thus we can locate the edge from parent $\left(v_{L}\right)$ to $u$ using $e$, in constant time. Let $x=y z$ be the partition of $x$ such that the path from parent $\left(v_{L}\right)$ to $v_{L}$ spells out $y$ and the path from $v_{L}$ to $u$ spells out $z$. Let $e^{\prime}$ be the out-going edge of $v_{L}$ in $\mathcal{T}_{2}$, that is the first edge in this path spelling out $z$. After the update to the growing suffix tree, the new edge from parent $\left(v_{L}\right)$ to $v_{L}$ is associated to $e$, and the new edge from $v_{L}$ to $u$ is associated to $e^{\prime}$. The edge $e^{\prime}$ can also be found by a level ancestor query from SA[PLV[SA $\left.\left.{ }^{-1}[k]\right]\right]$ on $\mathcal{T}_{2}$. See Figure 5 for illustration.

- If $L<R$, then leaf SA[NLV[SA $\left.\left.{ }^{-1}[k]\right]\right]$ will be the right neighbor of leaf $k$ in the updated suffix tree. This case can be treated in a similar manner as the afore-mentioned case.

We then associate the new leaf $k$ with the $\mathrm{SA}^{-1}[k]$-th entry of SA so that later, given $k$, we can access to this leaf on SA in $O(1)$ time. See also Figure 4 for a concrete example on how we insert a new leaf to the growing suffix tree.

Let us analyze the efficiency of our algorithm. Given SA, PLV and NLV can be constructed in $O(n)$ time [4]. Then, given a position $k$ in string $T^{R} \#$, we can access the leaves SA[PLV[SA $\left.\left.{ }^{-1}[k]\right]\right]$ and $\mathrm{SA}\left[\mathrm{NLV}\left[\mathrm{SA}^{-1}[k]\right]\right]$ of the full suffix tree $\mathcal{T}_{2}=\mathrm{STree}\left(T^{R} \#\right)$ in $O(1)$ time using SA, SA ${ }^{-1}$, PLV, and NLV arrays. The values of $L$ and $R$ can be computed in $O(1)$ time by two LCA queries on the full suffix tree $\mathcal{T}_{2}$. In each of the afore-mentioned cases, we perform at most two level ancestor queries on $\mathcal{T}_{2}$, using $O(1)$ time each. Thus it takes $O(1)$ time to insert a new leaf. Thus, it takes $O(1)$ time to insert a new leaf to the growing suffix tree $\mathcal{T}_{2}^{\prime}$. This completes the proof.

We now obtain the main result for this subsection:

Theorem 3. Given a string $T$ of length $n$ over an integer alphabet of size $\sigma=n^{O(1)}$, we can compute $\mathrm{SAGP}_{1}(T)$ in optimal $O\left(n+o c c_{1}\right)$ time and $O(n)$ space by using suffix trees, where occ ${ }_{1}=\left|\mathrm{SAGP}_{1}(T)\right|$.

Proof. We first compute the array $U$. Consider an arbitrary fixed $b$, and let $u u^{R}$ be a maximal palindrome stored in $U[b]$ whose center is $i=b+|u|-1$. Assume that we have a growing suffix tree $\mathcal{T}_{2}^{\prime}$ for string $T^{R}[n-b+1 . . n] \#$ which corresponds to the prefix $T[1 . . b]$ of $T$ of size $b$. We use a similar strategy as the suffix array based algorithms. For each position $2 n-b+2 \leq j \leq 2 n+1$ in string $T^{\prime}=T \$ T^{R} \#$, $1 \leq \mathrm{op}(j) \leq b-2$. We maintain the NMA data structure over the suffix tree $\mathcal{T}_{1}$ for string $T^{\prime}$ so that all the ancestors of the leaves whose corresponding suffixes start at positions $2 n-b+2 \leq j \leq 2 n+1$ are marked, and any other nodes in $\mathcal{T}_{1}$ remain unmarked at this step.

As in the suffix-array based algorithms, the task is to find the longest prefix $w^{R}$ of $T\left[i+\left|u^{R}\right|+1 . . n\right]$ such that $w$ occurs completely inside $T[1 . . b-2]=T[1 . . i-|u|-1]$. In so doing, we perform an NMA 

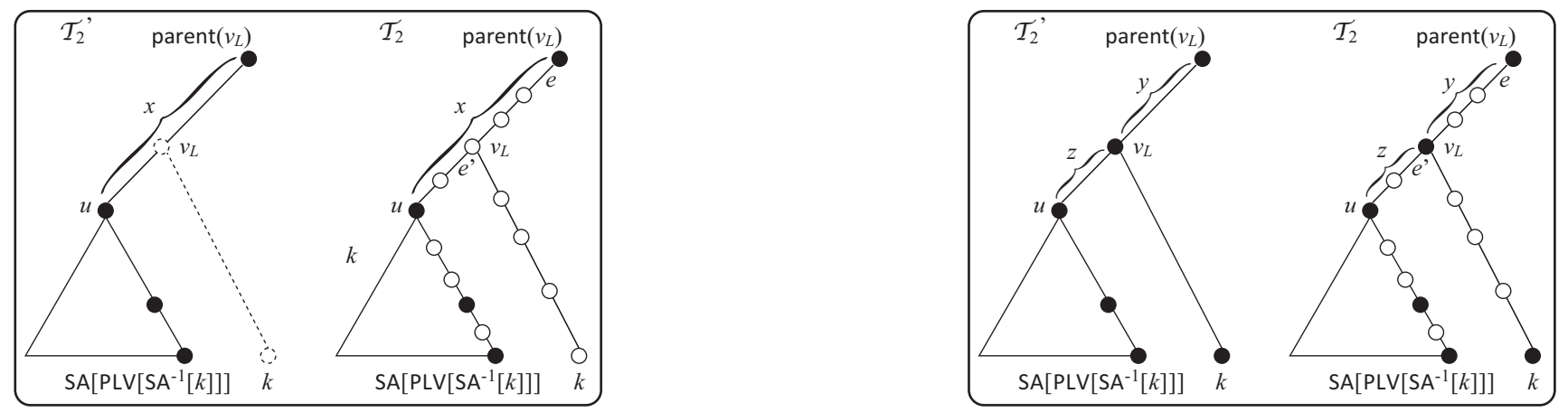

Figure 5: Left: The growing suffix tree $\mathcal{T}_{2}^{\prime}$ and the full suffix tree $\mathcal{T}_{2}$ before the update. Black nodes are marked nodes, and white ones are unmarked nodes. We find $v_{L}$ by an LCA query from the leaves for $\mathrm{SA}\left[\mathrm{PLV}\left[\mathrm{SA}^{-1}[k]\right]\right]$ and $k$ on $\mathcal{T}_{2}$, and find parent $\left(v_{L}\right)$ by an NMA query from $v_{L}$ on $\mathcal{T}_{2}$. We then find the edge $e$ on $\mathcal{T}_{2}$ by a level ancestor query from SA PLV $\left.\left[\mathrm{SA}^{-1}[k]\right]\right]$ on $\mathcal{T}_{2}$, and locate the edge from parent $\left(v_{L}\right)$ to $u$ on the growing tree $\mathcal{T}_{2}^{\prime}$ using a link from $e$. Right: The growing suffix tree $\mathcal{T}_{2}^{\prime}$ and the full suffix tree $\mathcal{T}_{2}$ after the update. Now the edge from parent $\left(v_{L}\right)$ to $v_{L}$ on $\mathcal{T}_{2}^{\prime}$ is associated to $e$, and the edge from $v_{L}$ to $u$ on $\mathcal{T}_{2}^{\prime}$ is associated to $e^{\prime}$.

query from the leaf $i+\left|u^{R}\right|+1$ of $\mathcal{T}_{1}$, and let $v$ be the answer to the NMA query. By the way how we have maintained the NMA data structure, it follows that $\operatorname{str}(v)=w^{R}$.

To obtain the occurrences of $w$ in $T[1 . . b-2]$, we switch to $\mathcal{T}_{2}^{\prime}$, and traverse the subtree rooted at $v$. Then, for any leaf $\ell$ in the subtree, $(i,|\operatorname{str}(v)|, b-\mathrm{op}(\ell),|u|)$ is a canonical longest SAGP for pivot $i$ (see also Figure 6).

After processing all the maximal palindromes in $U[b]$, we mark all unmarked ancestors of the leaf $2 n-b$ of $\mathcal{T}_{1}$ in a bottom-up manner, until we encounter the lowest ancestor that is already marked. This operation is a preprocessing for the maximal palindromes in $U[b+1]$, as we will be interested in the positions between 1 and op $(2 n-b)=b-1$ in $T$. In this preprocessing, each unmarked node is marked at most once, and each marked node will remain marked. In addition, we update the growing suffix tree $\mathcal{T}_{2}^{\prime}$ by inserting the new leaf for $T^{R}[n-b . . n] \#$.

We analyze the time complexity of this algorithm. Since all maximal palindromes in $U[b]$ begin at position $b$ in $T$, we can use the same set of marked nodes on $\mathcal{T}_{1}$ for all of those in $U[b]$. Thus, the total cost to update the NMA data structure for all $b$ 's is linear in the number of unmarked nodes that later become marked, which is $O(n)$ overall. The cost for traversing the subtree of $\mathcal{T}_{2}^{\prime}$ to find the occurrences of $w$ can be charged to the number of canonical longest SAGPs to output for each pivot, thus it takes $O\left(\right.$ occ $\left._{1}\right)$ time for all pivots. Updating the growing suffix tree $\mathcal{T}_{2}^{\prime}$ takes overall $O(n)$ time by Lemma 4 . What remains is how to efficiently link the new internal node introduced in the growing suffix tree $\mathcal{T}_{2}^{\prime}$, to its corresponding node in the static suffix tree $\mathcal{T}_{1}$ for string $T^{\prime}$. This can be done in $O(1)$ time using a similar technique based on LCA queries on $\mathcal{T}_{1}$, as in the proof of Lemma 4. Summing up all the above costs, we obtain $O\left(n+o c c_{1}\right)$ optimal running time and $O(n)$ working space.

Example 2. Let $T=$ acacabaabca and $T^{\prime}=$ acacabaabca $\$$ acbaabacaca\#, where $T^{\prime}=T \$ T^{R} \#$. First, we compute Pals and the array $U$. Assume we are now processing position $b=6$ in $T$, then $U[6]=\{(6,9)\}$, where $(6,9)$ represents the maximal palindrome $T[6 . .9]=$ baab. Thus we consider pivot $i=b+\lceil(9-6+$ 1) $/ 2\rceil-1=7$.

First, we construct the suffix tree $\mathcal{T}_{1}=\operatorname{STree}\left(T \$ T^{R} \#\right)$. Suppose that we have constructed $\mathcal{T}_{2}^{\prime}=$ $\mathrm{STree}\left(T^{R}[8 . .11] \#\right)$ and marked all ancestors of every leaf $v$ such that $19<v \leq 24$ in $\mathcal{T}_{1}$. In Figure 6 , we show interesting parts of $\mathcal{T}_{1}$ and $\mathcal{T}_{2}^{\prime}$. 


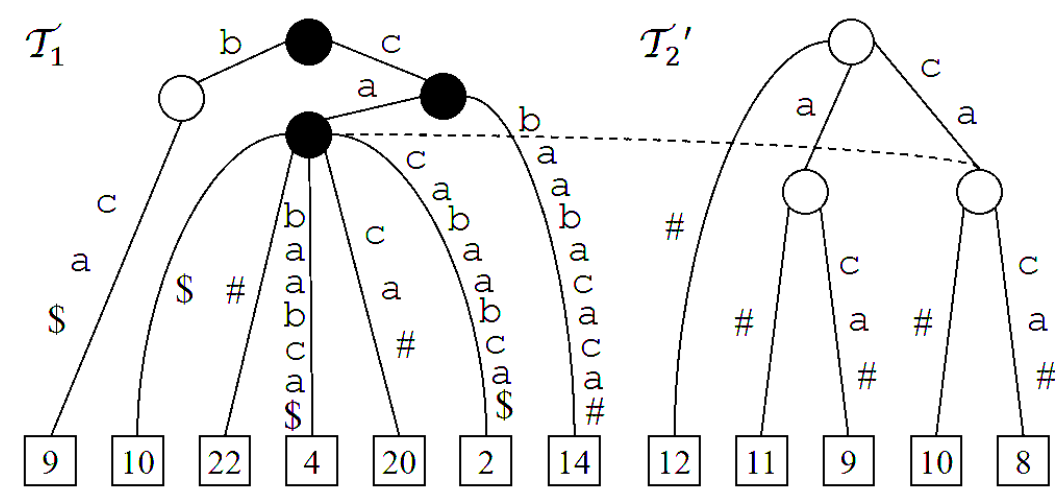

Figure 6: $\quad$ Showing interesting parts of $\mathcal{T}_{1}=\operatorname{STree}\left(T^{\prime}\right)$ and $\mathcal{T}_{2}^{\prime}=\operatorname{STree}\left(T^{R}[8 . .11] \#\right)$, where $T=$ acacabaabca, $T^{R}=$ acbaabacaca and $T^{\prime}=$ acacabaabca $\$$ acbaabacaca\#. In $\mathcal{T}_{1}$, we represent the marked internal nodes by black circles, the unmarked internal nodes by white circles, and the leaves by squares in which the numbers denote the beginning positions of the corresponding suffixes in the string. The dotted line represents the link between the node for string $\mathrm{c}$ in $\mathcal{T}_{1}$ and that in $\mathcal{T}_{2}^{\prime}$.

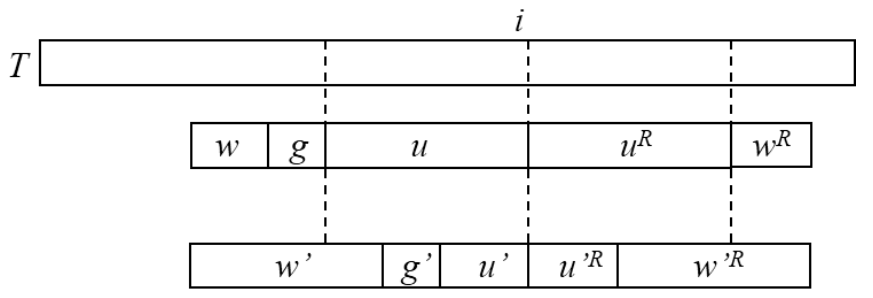

Figure 7: Illustration for Lemma 5.

To compute the longest $w$, we perform an $N M A$ query from the leaf $i+\left|u^{R}\right|+1=10$ of $\mathcal{T}_{1}$. As can be seen in Figure 6, we obtain the nearest marked node $v=\mathrm{NMA}_{\mathcal{T}_{1}}(10)$. Thus, we know that $w^{R}=\mathrm{ca}$. Next, we switch from the node $v$ of $\mathcal{T}_{1}$ to its corresponding node $v^{\prime}$ of $\mathcal{T}_{2}^{\prime}$ using a link between them. Then, we traverse the subtree rooted at $v^{\prime}$ and obtain all occurrences of $w^{R}$, namely $w^{R}=T^{R}[10 . .11]=T^{R}[8 . .9]=\mathrm{ca}$ at positions 10 and 8 in the reversed string $T^{R} \#$. Since $\mathrm{op}(10)=2$ and $\mathrm{op}(8)=4$, we obtain the canonical longest $\operatorname{SAGPs}(7,2,3,2)=\mathrm{ac}|\underline{\mathrm{aca}}| \mathrm{ba}|\mathrm{ab}| \mathrm{ca}$, and $(7,2,1,2)=\mathrm{ac}|\underline{\mathrm{a}}| \mathrm{ba}|\mathrm{ab}| \mathrm{ca}$ for pivot 7 .

\subsection{Computing $\mathrm{SAGP}_{2}(T)$ for type-2 positions}

In this subsection, we present an algorithm to compute $\mathrm{SAGP}_{2}(T)$ in a given string $T$, corresponding to the line 10 in Algorithm 1 .

Lemma 5. Every (not necessarily longest) SAGP for type-2 pivot $i$ must end at one of the positions between $i+2$ and $i+\operatorname{Pals}[i]$.

Proof. See Figure 7. By definition, it is clear that any SAGP for pivot $i$ must end at position $i+2$ or after that. Now, assume on the contrary that there exists a SAGP $w^{\prime} g^{\prime} u^{\prime} u^{\prime} w^{R}$ for pivot $i$ such that $i+\left|u^{\prime R} w^{\prime R}\right|>i+\left|u^{R}\right|$ (it ends after position $i+\left|u^{R}\right|$ ), where $u u^{R}$ is the maximal palindrome centered at position $i$. Recall that since $i$ is a type-2 position, we have $\left|u^{\prime}\right|<|u|$. Let $w^{R}$ be the suffix of $w^{\prime R}$ of size $\left|u^{\prime R} w^{\prime R}\right|-\left|u^{R}\right|$. Then, there exists a SAGP $w g u u^{R} w^{R}$ for pivot $i$ where $|g|=\left|g^{\prime}\right|$ and $u u^{R}$ is the maximal 


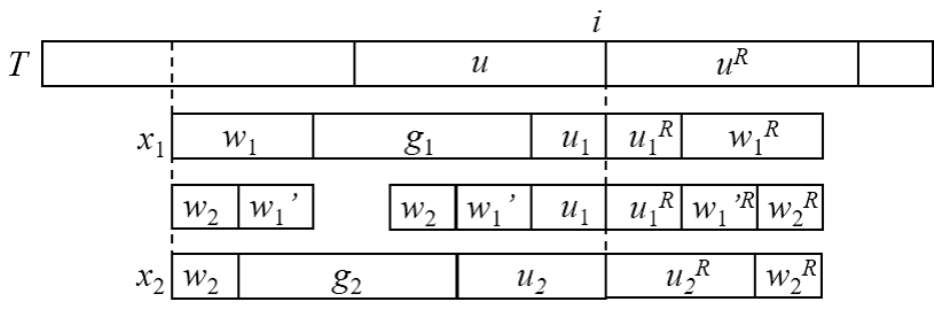

Figure 8: Illustration for Lemma 6.

palindrome centered at $i$. However, this contradicts that $i$ is a type-2 position. Hence, any SAGP for pivot $i$ must end at position $i+\left|u^{R}\right|$ or before that.

Lemma 6. For any type-2 position $i$ in string $T$, if $w g u u^{R} w^{R}$ is a canonical longest SAGP for pivot $i$, then $|w|=1$.

Proof. Let $x_{1}=w_{1} g_{1} u_{1} u_{1}^{R} w_{1}^{R}$ be a canonical longest SAGP for pivot $i$, and on the contrary, suppose that $\left|w_{1}\right| \geq 2$. See also Fig 8 . Then we can rewrite $w_{1}=w_{2} w_{1}^{\prime}$ for two non-empty strings $w_{2}$ and $w_{1}^{\prime}$. Let $u u^{R}$ be the maximal palindrome centered at $i$. Since the position $i$ is type-2, $u_{1}^{R} w_{1}^{R}$ is a prefix of $u^{R}$ by Lemma 5 , so that $w_{1} u_{1}$ is a suffix of $u$. Moreover, let $u_{2}=w_{1}^{\prime} u_{1}$ and $g_{2}$ be a string satisfying $g_{2} w_{1}^{\prime}=w_{1}^{\prime} g_{1}$. Then $x_{2}=w_{2} g_{2} \underline{u_{2}} u_{2}^{R} w_{2}^{R}=w_{2} g_{2} w_{1}^{\prime} u_{1} \underline{u_{2}^{R}} w_{2}^{R}=w_{2} \underline{g_{2}} w_{1}^{\prime} u_{1} u_{1}^{R} w_{1}^{\prime R} w_{2}^{R}=\underline{w_{2} w_{1}^{\prime}} g_{1} u_{1} u_{1}^{R} w_{1}^{\prime R} w_{2}^{R}=w_{1} g_{1} u_{1} u_{1}^{R} \underline{w_{1}^{\prime R} w_{2}^{R}}$ $=w_{1} g_{1} u_{1} u_{1}^{R} w_{1}^{R}=x_{1}$, that shows $x_{2}$ is also a SAGP for pivot $i$. Because armlen $\left(x_{2}\right)=\left|w_{2} u_{2}\right|=\left|w_{1} u_{1}\right|=$ $\operatorname{armlen}\left(x_{1}\right), x_{2}$ is also a longest SAGP for pivot $i$. Because $u_{2}=w_{1}^{\prime} u_{1}$ and $w_{1}^{\prime} \neq \varepsilon$, we have $\left|u_{2}\right|<\left|u_{1}\right|$, which contradicts that $x_{1}$ is a canonical longest SAGP for pivot $i$.

For every type-2 position $i$ in $T$, let $u=T[i . . i+$ Pals $[i]]$. By Lemma 6, any canonical longest SAGP is of the form $c g u u^{R} c$ for $c \in \Sigma$. For each $2 \leq k \leq \operatorname{Pals}[i]$, let $c_{k}=u^{R}[k]$, and let $u_{k}^{R}$ be the proper prefix of $u^{R}$ of length $k-1$. Now, observe that the largest value of $k$ for which LMost $\left[c_{k}\right] \leq i-\left|u_{k}\right|-1$ corresponds to a canonical longest SAGP for pivot $i$, namely, $c_{k} g_{k} u_{k} u_{k}^{R} c_{k}$ is a canonical longest SAGP for pivot $i$, where $g_{k}=T\left[\operatorname{LMost}\left[c_{k}\right]+1 . . i-\left|u_{k}\right|\right]$. In order to efficiently find the largest value of such, we consider a function findR $(t, i)$ defined by

$$
\text { findR }(t, i)=\min \{r \mid t \leq r<i, T[l]=T[r] \text { for } 1 \leq l<r\} \cup\{+\infty\} .
$$

Lemma 7. For any type-2 position $i$ in $T$, quadruple $(i, 1, r-\operatorname{LMost}[T[r]], i-r)$ represents a canonical longest $S A G P$ for pivot $i$, where $r=\operatorname{findR}(i-\operatorname{Pals}[i]+1, i) \neq \infty$. Moreover, its gap is the longest among all the canonical longest $S A G P$ s for pivot $i$.

Proof. See Figure 9, Let $t$ be the beginning position of $u$ in $T$, namely, $t=i-|u|+1$. Let $r=$ findR $(t, i)<$ $\infty$, and let $c=T[r]$. Then by definition of findR $(t, i)$, there exists $1 \leq l<r$ satisfying $T[l]=c$. Therefore, $x=(i, 1, r-l, i-r)$ is a SAGP for pivot $i$. Moreover, $x$ is canonical longest SAGP because $r$ is minimized, so that $\left|u_{1}\right|=i-r$ is maximized while $|w|$ is always 1 . Recall that LMost $[c]$ is the leftmost position $l$ satisfying $T[l]=c$. Hence, the gap size of the canonical longest $\operatorname{SAGP}(i, 1, r-\operatorname{LMost}[T[r]], i-r)$ is the longest.

By Lemma 7, we can compute a canonical longest SAGP for any type-2 pivot $i$ in $O(1)$ time, assuming that the function findR $(t, i)$ returns a value in $O(1)$ time. We define an array FindR of size $n$ by

$$
\text { FindR }[t]=\min \{r \mid t \leq r, \quad T[l]=T[r] \text { for } 1 \leq l<r\} \cup\{+\infty\},
$$




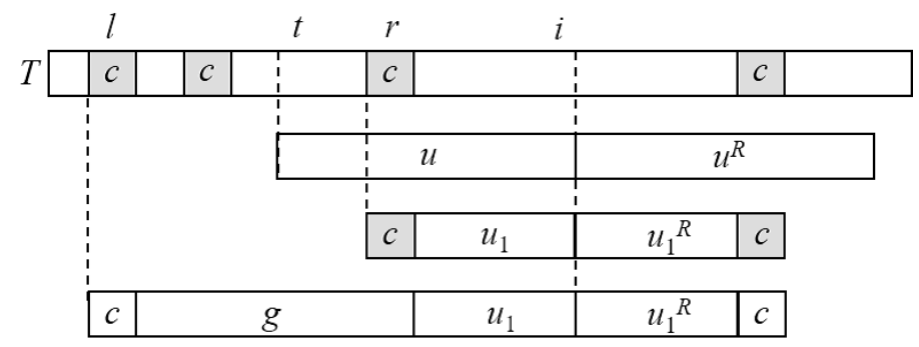

Figure 9: Illustration for Lemma 7.

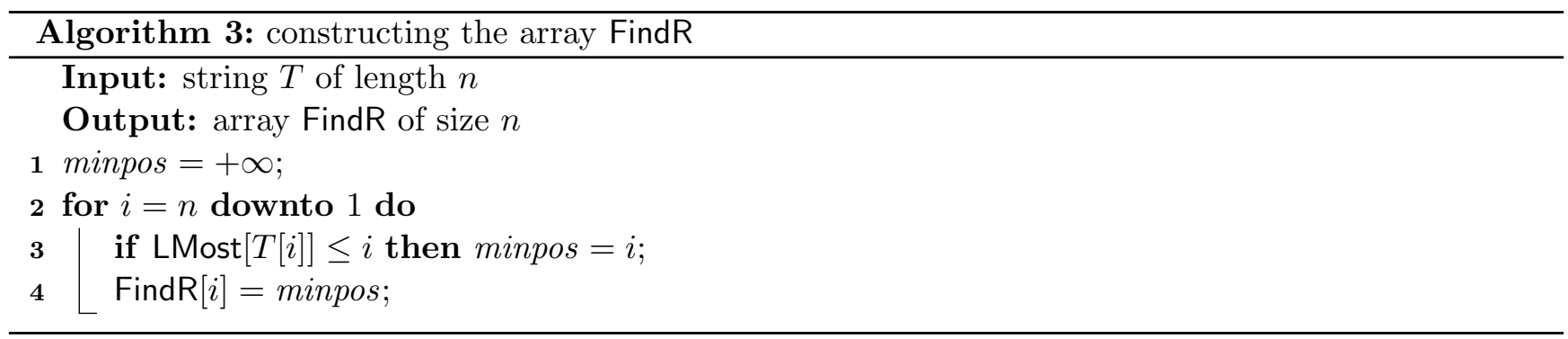

for $1 \leq t \leq n$. If the array FindR has already been computed, then findR $(t, i)$ can be obtained in $O(1)$ time by findR $(t, i)=$ FindR $[t]$ if FindR $[t]<i$, and $+\infty$ otherwise.

Algorithm 3 shows a pseudo-code to compute FindR. Table 1 shows an example.

Lemma 8. Algorithm 3 correctly computes the array FindR in $O(n)$ time and space.

Proof. The correctness of the computation of LMost is obvious. For each $i$, we maintaining invariant minpos $=\min \{r \mid i \leq t, T[l]=T[r]$ for some $1 \leq l<r\}$. minpos can be maintained by updating it if $i$ is not the leftmost occurrence position of $T[i]$. Since the definition of FindR $[i]$ is the same as minpos for each $i$, FindR $[i]$ can be computed by assigning minpos to FindR $[i]$.

By Lemma 8, we can compute $\mathrm{SAGP}_{2}(T)$ for type-2 positions as follows.

Theorem 4. Given a string $T$ of length $n$ over an integer alphabet of size $n^{O(1)}$, we can compute $\mathrm{SAGP}_{2}(T)$ in $O\left(n+o c c_{2}\right)$ time and $O(n)$ space, where occ $_{2}=\left|\mathrm{SAGP}_{2}(T)\right|$.

Proof. For a given $T$, we first compute the array FindR by Algorithm3. The correctness of the computation of NextPos is obvious. By Lemma 7, we can get a canonical longest SAGP $x_{1}=\left(i, 1,\left|g_{1}\right|\right.$, Pals $\left.[i]-r\right)$ if Pals $[i]-r \geq 1$, in $O(1)$ time by referring to LMost and FindR. Note that $x_{1}$ is the one whose gap $\left|g_{1}\right|$ is the longest. Let $b_{1}=i-\operatorname{Pals}[i]+r-\left|g_{1}\right|$ be the beginning position of $x_{1}$ in $T$. Then the next shorter canonical longest SAGP for the same pivot $i$ begins at position $b_{2}=\operatorname{NextPos}\left[b_{1}\right]$. By repeating this process $b_{j+1}=\operatorname{NextPos}\left[b_{j}\right]$ while the gap size $\left|g_{j}\right|=i-\operatorname{Pals}[i]+r-b_{j}$ is positive, we obtain all the canonical longest SAGPs for pivot $i$. Overall, we can compute all canonical longest SAGPs for all pivots in $T$ in $O\left(n+o c c_{2}\right)$ time. The space requirement is clearly $O(n)$.

We now have the main theorem from Theorem 3, Lemma 2, Lemma 3, and Theorem 4 as follows.

Theorem 5. Given a string $T$ of length $n$ over an integer alphabet of size $n^{O(1)}$, Algorithm 1 can compute $\operatorname{SAGP}(T)$ in optimal $O(n+o c c)$ time and $O(n)$ space, where occ $=|\operatorname{SAGP}(T)|$. 
Table 1: Arrays LMost, NextPos, and FindR for a string $T=$ dbbaacbcbad. For the sake of understanding, we also provide the values of $\min _{\text {out }}$ and $\min _{\text {in }}$ in the $i$-th loop of Algorithm3. These values are computed from right to left.

\begin{tabular}{|c|c|}
\hline & LMost \\
\hline $\mathrm{a}$ & 4 \\
$\mathrm{~b}$ & 2 \\
$\mathrm{c}$ & 6 \\
$\mathrm{~d}$ & 1 \\
\hline
\end{tabular}

\begin{tabular}{|c|c|c|c|c|c|c|c|c|c|c|c|}
\hline & 1 & 2 & 3 & 4 & 5 & 6 & 7 & 8 & 9 & 10 & 11 \\
\hline$T$ & $\mathrm{~d}$ & $\mathrm{~b}$ & $\mathrm{~b}$ & $\mathrm{a}$ & $\mathrm{a}$ & $\mathrm{c}$ & $\mathrm{b}$ & $\mathrm{c}$ & $\mathrm{b}$ & $\mathrm{a}$ & $\mathrm{d}$ \\
\hline NextPos & 11 & 3 & 7 & 5 & 10 & 8 & 9 & $\infty$ & $\infty$ & $\infty$ & $\infty$ \\
\hline FindR & 3 & 3 & 3 & 5 & 5 & 7 & 7 & 8 & 9 & 10 & 11 \\
\hline
\end{tabular}

Table 2: Running times (in milli-sec.) on randomly generated strings of length 10000, 50000, and 100000 with $|\Sigma|=10$.

\begin{tabular}{|r|r|r|r|r|r|}
\hline \multicolumn{1}{c|}{$n$} & \multicolumn{1}{c|}{ Naïve } & Traverse & RB tree & vEB tree & Y-fast trie \\
\hline \hline 10000 & 247.2 & 3.8 & 6.3 & 85.7 & 11.7 \\
50000 & 7661.0 & 18.6 & 37.2 & 128.9 & 62.6 \\
100000 & 32933.2 & 38.7 & 80.3 & 191.9 & 133.7 \\
\hline
\end{tabular}

\section{Experiments}

In this section, we show some experimental results which compare performance of our algorithms for computing $\operatorname{SAGP}_{1}(T)$. We implemented the naïve quadratic-time algorithm (Naïve), the simple quadratictime algorithm which traverses suffix arrays (Traverse), and three versions of the algorithm based on suffix array and predecessor/successor data structure, each employing red-black trees (RB tree), Y-fast tries (Yfast trie), and van Emde Boas tree ${ }^{1}$ (vEB tree), as the predecessor/successor data structure.

We implemented all these algorithms with Visual C++ 12.0 (2013), and performed all experiments on a PC (Intel@) Xeon CPU W3565 3.2GHz, 12GB of memory) running on Windows 7 Professional. In each problem, we generated a string randomly and got the average time for ten times attempts.

We tested all programs on strings of lengths 10000, 50000, and 100000, all from an alphabet of size $|\Sigma|=10$. Table 2 shows the results. From Table 2, we can confirm that Traverse is the fastest, while Naive is by far the slowest. We further tested the algorithms on larger strings with $|\Sigma|=10$. In this comparison, we excluded Naïve as it is too slow. The results are shown in Figure 10. As one can see, Traverse was the fastest for all lengths. We also conducted the same experiments varying alphabet sizes as 2, 4, and 20, and obtained similar results as the case of alphabet size 10 .

To verify why Traverse runs fastest, we measured the average numbers of suffix array entries which are traversed, per pivot and output (i.e., canonical longest SAGP). Figure 11 shows the result. We can observe that although in theory $O(n)$ entries can be traversed per pivot and output for a string of length $n$, in both cases the actual number is far less than $n$ and grows very slowly as $n$ increases. This seems to be the main reason why Traverse is faster than RB tree, vEB tree, and Y-fast trie which use sophisticated but also complicated predecessor/successor data structures.

\footnotetext{
${ }^{1}$ We modified a van Emde Boas tree implementation from https://code.google.com/archive/p/libveb/ so it works with Visual $\mathrm{C}++$.
} 


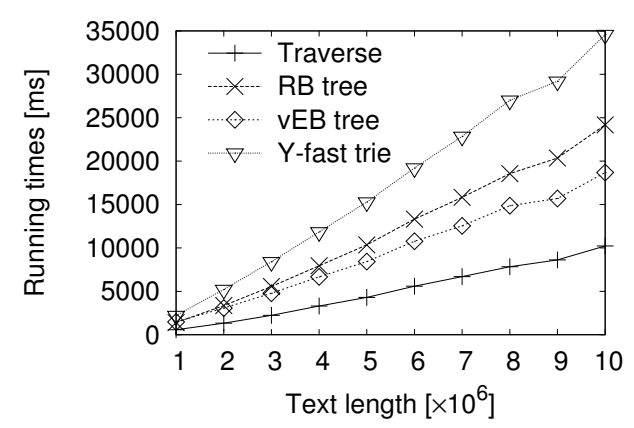

Figure 10: Running times (in milli-sec.) on randomly generated strings of length from 1000000 to 10000000 with $|\Sigma|=10$.

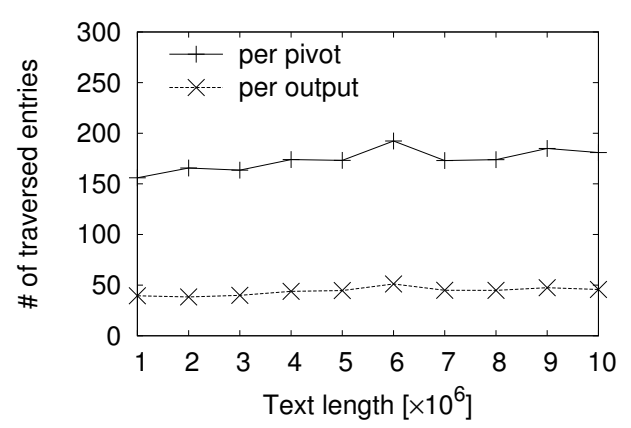

Figure 11: Average numbers of traversed entries of suffix array per pivot and per output on randomly generated strings.

\section{Conclusions and future work}

We proposed several algorithms to compute longest single-arm-gapped palindromes (SAGPs) for all pivots in a given string $T$ of length $n$. For the type- 1 longest SAGPs, we presented an $O\left(\left(n+o c c_{1}\right) \log \log n\right)$-time algorithm which is based on a suffix array and a dynamic predecessor/successor data structure, where $o c c_{1}$ is the number of type- 1 longest SAGPs to output. We also presented an $O\left(n+o c c_{1}\right)$-time algorithm based on the suffix tree. For the type- 2 longest SAGPs, we proposed an $O\left(n+o c c_{2}\right)$-time algorithm, where $o c c_{2}$ is the number of type-2 longest SAGPs to output. Combining the last two results, we obtained an optimal $O(n+o c c)$-time algorithm for computing all longest SAGPs, where $o c c=o c c_{1}+o c c_{2}$ is the total number of outputs. We performed experiments to compare practical performances of our algorithms for finding type-1 longest SAGPs; the naïve algorithm, the $O\left(n^{2}\right)$-time suffix array based algorithm, and the improved suffix array based algorithm with several kinds of predecessor/successor data structures.

Our future work includes the following: Is there a linear $O\left(n+o c c_{1}\right)$-time algorithm for finding type-1 longest SAGPs using a suffix array (plus some auxiliary arrays), rather than a suffix tree? Our current best suffix-array-based algorithm uses $O\left(\left(n+o c c_{1}\right) \log \log n\right)$ time. Another question is how many longest SAGPs can be contained in a string. Finding non-trivial upper bound and/or lower bound for occ remains open.

\section{Acknowledgments}

The research of Shintaro Narisada, Kazuyuki Narisawa, and Ayumi Shinohara was supported by JSPS KAKENHI Grant Numbers JP15H05706, JP24106010, and ImPACT Program Tough Robotics Challenge of Japan Science and Technology Agency. The research of Diptarama Hendrian is supported by Tohoku University Division for Interdisciplinary Advance Research and Education. The research of Shunsuke Inenaga was supported by JSPS KAKENHI Grant Numbers JP26280003 and JP17H01697.

The authors thank anonymous referees for their useful suggestions for improving the quality of the paper and for pointing out some errors in the previous version. 


\section{References}

[1] Alberto Apostolico, Dany Breslauer, and Zvi Galil. Parallel detection of all palindromes in a string. Theor. Comput. Sci., 141(1-2):163-173, 1995.

[2] Michael A. Bender and Martín Farach-Colton. The LCA problem revisited. In LATIN, pages 88-94, 2000.

[3] Michael A. Bender and Martin Farach-Colton. The Level Ancestor Problem simplified. Theor. Comput. Sci., 321(1):5-12, 2004.

[4] Maxime Crochemore, Lucian Ilie, Costas S. Iliopoulos, Marcin Kubica, Wojciech Rytter, and Tomasz Walen. Computing the longest previous factor. Eur. J. Comb., 34(1):15-26, 2013.

[5] Maxime Crochemore, Costas S. Iliopoulos, Marcin Kubica, Wojciech Rytter, and Tomasz Waleń. Efficient algorithms for two extensions of LPF table: The power of suffix arrays. In SOFSEM 2010: Theory and Practice of Computer Science, volume 5901 LNCS, pages 296-307, 2010.

[6] Xavier Droubay and Giuseppe Pirillo. Palindromes and Sturmian words. Theor. Comput. Sci., 223(1-2):73-85, 1999.

[7] Marius Dumitran, Paweł Gawrychowski, and Florin Manea. Longest gapped repeats and palindromes. Discrete Mathematics \& Theoretical Computer Science, 19(4), 2017.

[8] Marius Dumitran and Florin Manea. Longest gapped repeats and palindromes. In Mathematical Foundations of Computer Science 2015, volume 9234, pages 205-217, 2015.

[9] Martin Farach-Colton, Paolo Ferragina, and S. Muthukrishnan. On the sorting-complexity of suffix tree construction. J. ACM, 47(6):987-1011, 2000.

[10] Yuta Fujishige, Michitaro Nakamura, Shunsuke Inenaga, Hideo Bannai, and Masayuki Takeda. Finding gapped palindromes online. In IWOCA 2016, pages 191-202, 2016.

[11] Pawel Gawrychowski, Tomohiro I, Shunsuke Inenaga, Dominik Köppl, and Florin Manea. Tighter bounds and optimal algorithms for all maximal $\alpha$-gapped repeats and palindromes — Finding all maximal $\alpha$-gapped repeats and palindromes in optimal worst case time on integer alphabets. Theory Comput. Syst., 62(1):162-191, 2018.

[12] Amy Glen, Jacques Justin, Steve Widmer, and Luca Q. Zamboni. Palindromic richness. Eur. J. Comb., 30(2):510-531, 2009.

[13] Dan Gusfield. Algorithms on Strings, Trees, and Sequences. Cambridge University Press, 1997.

[14] Tomohiro I and Dominik Kppl. Improved upper bounds on all maximal $\alpha$-gapped repeats and palindromes. Theoretical Computer Science, 753:1-15, 2019.

[15] Juha Kärkkäinen, Peter Sanders, and Stefan Burkhardt. Linear work suffix array construction. J. ACM, 53(6):918-936, 2006.

[16] Toru Kasai, Gunho Lee, Hiroki Arimura, Setsuo Arikawa, and Kunsoo Park. Linear-time longestcommon-prefix computation in suffix arrays and its applications. In CPM 2001, pages 181-192, 2001. 
[17] Roman Kolpakov and Gregory Kucherov. Searching for gapped palindromes. Theor. Comput. Sci., 410(51):5365-5373, 2009.

[18] Glenn K. Manacher. A new linear-time on-line algorithm for finding the smallest initial palindrome of a string. J. ACM, 22(3):346-351, 1975.

[19] Udi Manber and Eugene W. Myers. Suffix arrays: A new method for on-line string searches. SIAM J. Comput., 22(5):935-948, 1993.

[20] Shintaro Narisada, Diptarama, Kazuyuki Narisawa, Shunsuke Inenaga, and Ayumi Shinohara. Computing longest single-arm-gapped palindromes in a string. In SOFSEM, pages 375-386, 2017.

[21] Ya-Zhou Shi, Feng-Hua Wang, Yuan-Yan Wu, and Zhi-Jie Tan. A coarse-grained model with implicit salt for RNAs: Predicting 3D structure, stability and salt effect. The Journal of Chemical Physics, 141(10):105102, 2014.

[22] Peter van Emde Boas. Preserving order in a forest in less than logarithmic time. In FOCS, pages 75-84, 1975.

[23] Peter Weiner. Linear pattern matching algorithms. In 14th Annual Symposium on Switching and Automata Theory, pages 1-11, 1973.

[24] Jeffery Westbrook. Fast incremental planarity testing. In ICALP, pages 342-353, 1992.

[25] D. E. Willard. Log-logarithmic worst-case range queries are possible in space $\Theta(N)$. Information Processing Letters, 17:81-84, 1983. 